OPEN ACCESS

Edited by:

Sandro Mussa-Ivaldi,

Northwestern University,

United States

Reviewed by:

Xin Liu,

University of California, San Diego,

United States

Senentxu Lanceros-Mendez,

Basque Center for Materials, Applications and Nanostructures,

Spain

${ }^{*}$ Correspondence: Manuele Gor m.gori@unicampus.it

Specialty section:

This article was submitted to Bionics and Biomimetics,

a section of the journal

Frontiers in Bioengineering and

Biotechnology

Received: 26 January 2021 Accepted: 27 April 2021

Published: 25 May 2021

Citation:

Gori M, Vadalà G, Giannitelli SM,

Denaro V and Di Pino G (2021)

Biomedical and Tissue Engineering

Strategies to Control Foreign Body

Reaction to Invasive Neural

Electrodes.

Front. Bioeng. Biotechnol. 9:659033.

doi: 10.3389/fbioe.2021.659033

\section{Biomedical and Tissue Engineering Strategies to Control Foreign Body Reaction to Invasive Neural Electrodes}

\author{
Manuele Gori1,2*, Gianluca Vadalà̀ ${ }^{1}$, Sara Maria Giannitelli³, Vincenzo Denaro ${ }^{1}$ and \\ Giovanni Di Pino ${ }^{4}$
}

${ }^{1}$ Laboratory for Regenerative Orthopaedics, Department of Orthopaedic Surgery and Traumatology, Università Campus Bio-Medico di Roma, Rome, Italy, ${ }^{2}$ Institute of Biochemistry and Cell Biology (IBBC) - National Research Council (CNR), Rome, Italy, ${ }^{3}$ Laboratory of Tissue Engineering, Department of Engineering, Università Campus Bio-Medico di Roma, Rome, Italy, ${ }^{4} \mathrm{NeXT}$ : Neurophysiology and Neuroengineering of Human-Technology Interaction Research Unit, Università Campus Bio-Medico di Roma, Rome, Italy

Neural-interfaced prostheses aim to restore sensorimotor limb functions in amputees. They rely on bidirectional neural interfaces, which represent the communication bridge between nervous system and neuroprosthetic device by controlling its movements and evoking sensory feedback. Compared to extraneural electrodes (i.e., epineural and perineural implants), intraneural electrodes, implanted within peripheral nerves, have higher selectivity and specificity of neural signal recording and nerve stimulation. However, being implanted in the nerve, their main limitation is represented by the significant inflammatory response that the body mounts around the probe, known as Foreign Body Reaction (FBR), which may hinder their rapid clinical translation. Furthermore, the mechanical mismatch between the consistency of the device and the surrounding neural tissue may contribute to exacerbate the inflammatory state. The FBR is a non-specific reaction of the host immune system to a foreign material. It is characterized by an early inflammatory phase eventually leading to the formation of a fibrotic capsule around intraneural interfaces, which increases the electrical impedance over time and reduces the chronic interface biocompatibility and functionality. Thus, the future in the reduction and control of the FBR relies on innovative biomedical strategies for the fabrication of next-generation neural interfaces, such as the development of more suitable designs of the device with smaller size, appropriate stiffness and novel conductive and biomimetic coatings for improving their long-term stability and performance. Here, we present and critically discuss the latest biomedical approaches from material chemistry and tissue engineering for controlling and mitigating the FBR in chronic neural implants.

Keywords: neural electrodes, foreign body reaction, coatings, biomaterials, hydrogel, tissue engineering, microfluidics, nanofabrication techniques 


\section{INTRODUCTION}

Since scientists started to invasively study the function of the central nervous system (CNS) and peripheral nervous system (PNS), single electrodes, and later on electrode arrays, have been implanted to record neuronal activity and to stimulate single or groups of neurons to artificially induce their activation, in light of decoding their functions.

Once study protocols moved from acute tests to chronic implantations and the safety of implants performed in primates suggested the possibility to move to studies in humans, a further possible application of invasive neural electrodes, beside that to investigate neuronal functions, became concrete. Electrodes started to be employed to decode subject motor intention and, bypassing neural or osteo-muscular lesions, to artificially interface the nervous system to the external environment.

When this happened, neural interfaces -often named brain to computer or to machine interfaces- and the field of neuroprosthetics were born. Depending on the site and the subject receiving the implant, electrodes can also be interfaced with sensory area and fibers and, by relaying afferent streams of information, convey artificial sensory feedback.

Insofar, some applications for stimulating neural electrodes, particularly deep brain stimulation (DBS) and cochlear implants, have gained the maturity to be commonly applied in clinical practice. Other applications targeting a more spatially-selective information exchange, such as cortical or peripheral nerve implants, are very-promising, yet still in a developmental phase. Their not-complete maturity is mostly due to the lack of longlasting stability of their performance over time, mainly because of the reaction that the body mounts around them. This factor hampers to a less extent cochlear electrodes, because they do not penetrate the neural structures, and DBS, because these electrodes do not need to achieve the level of stimulation selectivity needed by information exchange. The long-term functionality and longevity of cochlear implants and deep brain stimulators have already been widely demonstrated (Deuschl et al., 2006; Woeppel et al., 2017).

Contrarily, the use of invasive multichannel electrodes, implanted within stump peripheral nerves to control cybernetic hand prostheses, is an application field of neural interfaces where electrodes should achieve an intimate contact with neural fibers required to reach a reliable information transmission, and where implantable solutions seem to favor exchange selectivity.

Since peripheral nerves contain both motor and sensory fibers, peripheral nerve electrodes can achieve proper bidirectional communication through the use of a single device by stimulating afferent axons (Xavier and Jaume, 2014).

Regained sensory feedback from hand prosthesis has the potential to improve motor control (Valle et al., 2018; Zollo et al., 2019), discrimination abilities (Raspopovic et al., 2014), and to reverse aberrant brain plasticity triggered by the amputation (Rossini et al., 2010; Di Pino et al., 2012, 2014; Ferreri et al., 2014; Serino et al., 2017).

Unfortunately, the standard control systems of prosthetic limbs rely on surface electromyogram, which is mainly limited by problems of high latency, as well as low specificity and robustness in long-term implants (Anderson and Weir, 2019). Although some of the current peripheral nerve interfaces can shorten latency and provide single axon specificity, their performances tend to degrade with time due to the biological response of the organism to the electrode, which is triggered by the damage provoked by the implant procedure itself (Anderson and Weir, 2019). The body tends to insulate and exclude the foreign material from the surrounding microenvironment, leading to scar tissue growth around the device that is made of a fibrous capsule.

In the conductive surface, the dielectric constant, dissipation factor and dielectric loss factor rise with the increase of the capsule thickness. The increase of the electrical impedance is proportional to the development of the fibrotic tissue, which determines difficulties to distinguish the signal from background noise (Szostak et al., 2017) and, eventually, the drop of stimulation and registration capacities (Guadarrama-Santana and Garcia-Valenzuela, 2007; Jayamani et al., 2014).

The immune-mediated response responsible for the capsule growth is known as Foreign Body Reaction (FBR). FBR reduction over time is probably the main challenge for future neural electrode applications in neuroprosthetics to extend the reliability of the interface (Lotti et al., 2017).

The aim of this review is to analyze the latest tissue engineering strategies and biomedical approaches for controlling and evading FBR around implantable interfaces.

Although the FBR process can occur in any living tissue implanted with foreign material, such as molecularly engineered surfaces and medical devices (Anderson et al., 1999; Luttikhuizen et al., 2006), we will restrict our field of investigation and focus the review toward intraneural electrode applications to interface robotic prosthetic limbs.

We analyze factors supposed to be the main causes of acute and chronic neural tissue reactions, such as scarce biocompatibility, excessive size, poor flexibility, reduced electrical properties, low compliance, mechanical mismatch and micromotion.

Finally, we examine the shortcomings of current electrodeproducing technologies and discuss possible cutting-edge solutions for the development of promising alternatives to the present intraneural interfaces. Strategies and technologies analyzed in light of the specific application we pursue could be potentially tailored to any electrode inserted in the CNS or PNS, and interfaced with different artificial devices.

\section{MOLECULAR MECHANISMS AND CELLULAR COMPONENTS OF THE FBR}

In a living tissue or a nerve, any implantation of foreign material, including advanced biomaterials that surround an invasive electrode, triggers an unbalanced biological reaction (i.e., characterized by scarce wound healing and chronic inflammatory state) of the non-specific immune system, known as FBR, which is the natural protection mechanism of the host to the foreign body (Anderson et al., 1999; Luttikhuizen et al., 2006). This complex host reaction (Figure 1) consists in a sequential and orderly cascade of molecular events that involves adhesive blood and 


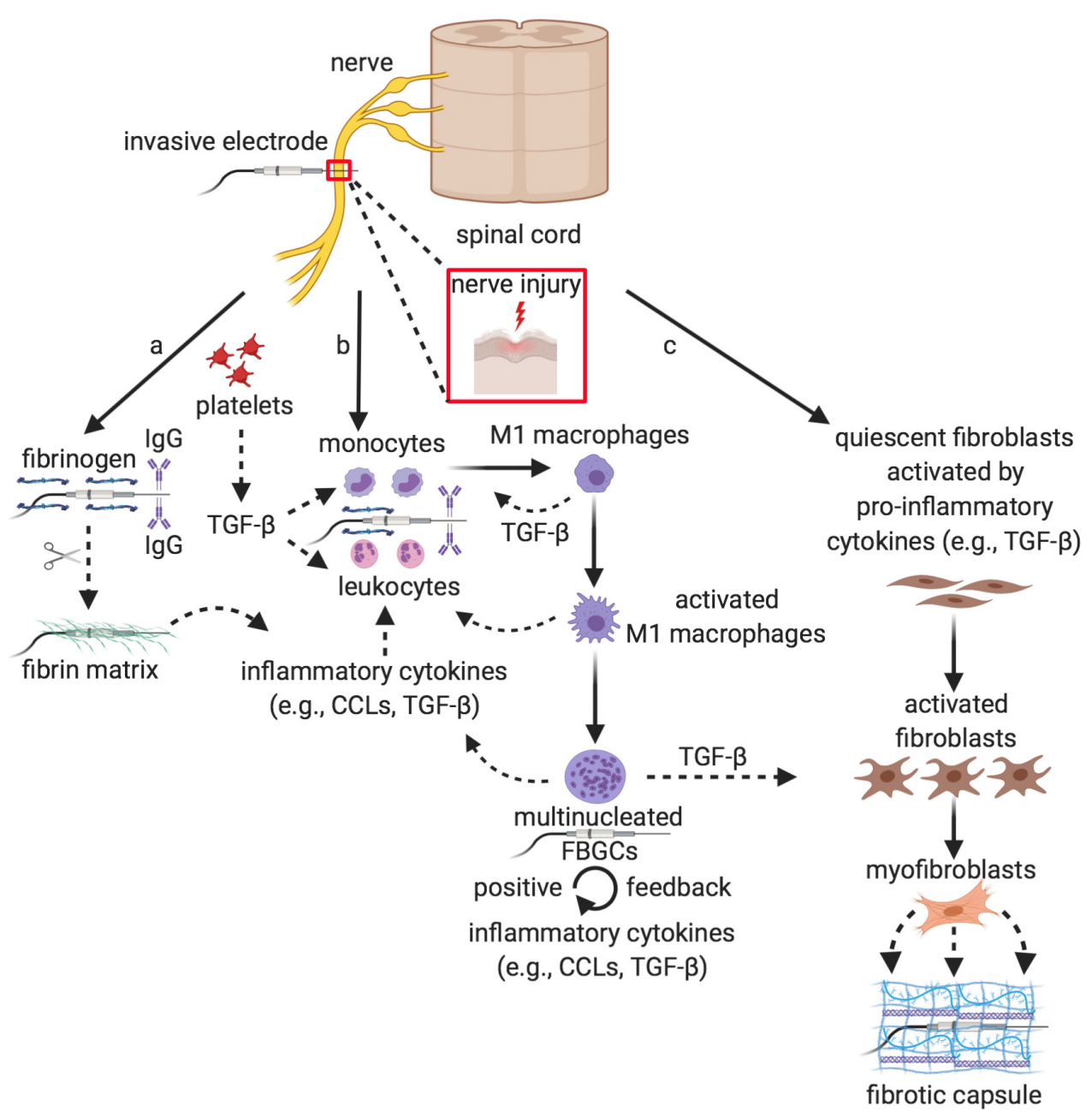

FIGURE 1 | Onset, progression and resolution of the Foreign Body Reaction. Sequence of cellular events of the non-specific immune response elicited by the biomaterial surrounding the invasive electrode implanted into the nervous tissue, which is perceived as a nerve injury: (a) onset, similarly to the wound healing, the adsorption of blood and plasma proteins [in particular, fibrinogen and antibodies (lgG), which will be recognized by the white blood cells of the immune system, and the complement system providing specific binding sites and chemoattractants for circulating leukocytes and monocytes] to the surface of the implant leads to the second step of the process, (b) the progression of the FBR, during which leukocyte and monocyte extravasation that is due to the influence of various chemokines, such as TGF- $\beta$, promotes their attraction and adhesion to the electrode surface. Recruited monocytes differentiate into activated M1 macrophages that fuse together into multinucleated FBGCs, which carry out multiple functions including: the increase of the inflammatory response both through a positive feedback mechanism (mainly via additional TGF- $\beta$ production) and through the recruitment of further monocytes and macrophages, the digestion of the electrode surface while promoting the recruitment of the fibroblasts and their activation to myofibroblasts in the last step of the process, (c) the resolution of the FBR, during which the myofibroblasts secrete the different ECM components around the implant that are responsible for the formation of the fibrotic capsule, which ultimately isolates the electrode from the surrounding tissue. IgG, immunoglobulin G; CCLs, CC chemokines; TGF- $\beta$, transforming growth factor $\beta$; FBGCs, foreign body giant cells. Created with BioRender.com.

plasma proteins, tissue and infiltrated inflammatory cells, and inflammatory cytokines.

The first step (onset), which is similar to wound healing, upon foreign body implantation is the adsorption of blood and plasma proteins, such as fibrinogen, fibronectin, albumin and antibodies to the implant surface (Andrade and Hlady, 1987; Jenney and Anderson, 2000). The type of the proteins adsorbed and the progression of the FBR depend on the surface shape, chemistry composition and charge (Tang and Eaton, 1993; Hunt et al., 1996; Thull, 2002). In the second step (progression), the adsorbed protein layer and its composition in turn promote monocyte and leukocyte extravasation, attraction and adhesion to the surface, along with the activation of the coagulation cascade (Richardson et al., 1976; Smiley et al., 2001; Szaba and Smiley, 2002).

Fibrinogen is hydrolyzed to fibrin that creates a sort of matrix able to attract circulating leukocytes and local macrophages around the implanted surface under the chemoattractive influence of different chemokines (Tang et al., 1998; Smiley et al., 2001; Tao and Kobzik, 2002; Lishko et al., 2004). At the onset of the FBR and during its progression, circulating platelets first and macrophages then secrete transforming growth factor $\beta$ (TGF- $\beta$ ). This pivotal cytokine serves as chemoattractant 
and activator of monocytes, besides being responsible for the continuum of the inflammation and its exacerbation until fibrosis (DiPietro et al., 1998; Crowe et al., 2000). Leukocytes express and secrete a series of other inflammatory cytokines, such as CCL2, CCL3, CCL5, which are the principal players involved in the recruitment of blood-borne monocytes and local macrophages in the foreign body microenvironment (DiPietro et al., 1998; Hancock et al., 2000; Ono et al., 2003; Armstrong et al., 2004). Afterward, extravasated monocytes differentiate to macrophages that, once activated under the stimulation of activated $\mathrm{T}$ cells, fuse together to form multinucleated foreign body giant cells (FBGCs). FBGCs start releasing further inflammatory cytokines, thus boosting the inflammatory response through a mechanism of positive feedback, giving rise to a chronic inflammation (Anderson, 2000; Kyriakides et al., 2004). This cell recruitment from the bloodstream is allowed by vasodilatation and increase of vessels permeability, which is induced by the platelet release of the angiogenic cytokine vascular endothelial growth factor (VEGF) (Banks et al., 1998; Ferrara et al., 2003). The biological activity of the FBGCs represents a hallmark of the FBR, as it is aimed to protect implanted tissue against the foreign body, mediating its surface damage and digestion through the release of various proteases and acids (Kyriakides et al., 2004). In the last step of the process (resolution), macrophages play a key role via the production of TGF- $\beta$. This multifunctional cytokine has a paramount importance as it will stimulate the fibroblast-mediated extracellular matrix (ECM) production, while reducing at the same time inflammation (Bellingan, 1996; Ashcroft, 1999). Thus, the role of the recruited macrophages is to promote further monocyte and macrophage recruitment and to stimulate the growth and differentiation of quiescent fibroblasts to myofibroblasts. Myofibroblasts are eventually responsible for the massive production and secretion of ECM components, including collagen I, collagen III, fibronectin and proteoglycans that give rise to the dense fibrotic capsule around the implanted electrode (Luttikhuizen et al., 2006; Anderson et al., 2008; Ward, 2008). In the very final stage of the process, the capsule becomes impermeable to the nonspecific immune system and to many chemicals, including some therapeutic inhibitors of inflammation, and responsible for the augmentation of the electric impedance and progressive isolation of the implanted device, impairing its long-term functionality (Anderson et al., 1999, 2008; Luttikhuizen et al., 2006).

\section{INTRANEURAL VS. EXTRANEURAL ELECTRODES IN FBR}

To interface with a peripheral nerve invasive intraneural and extraneural electrodes can be employed. Among intraneural electrodes, the most used are Multielectrode arrays (MEAs), Longitudinal Intra-Fascicular Electrodes (LIFEs) and Transverse Intrafascicular Multichannel Electrode (TIME) (Yoshida and Stein, 1999; Branner et al., 2004; Badia et al., 2011; Yildiz et al., 2020). The extraneural electrodes developed to interface with peripheral nerve are cuff electrodes (Navarro et al., 2001; OrtizCatalan et al., 2013) and Flat Interface Nerve Electrodes (FINEs) (Tyler and Durand, 2002; Freeberg et al., 2020).

Intraneural electrodes should offer a better signal-to-noise ratio during neural recording and the reduced current intensity necessary to reach the appropriate nerve stimulation (Navarro et al., 2005). Nonetheless, being implanted within the nerve, these interfaces are traumatic for the surrounding tissue triggering an early inflammatory response caused by the injury of the vascularized connective tissue. Indeed, as the electrode proximity to the nerve increases, a higher selectivity of neural recording of the signal and stimulation can be obtained. However, the formation of the fibrotic capsule around the interface reduces recording and stimulation long-term stability compared to extraneural electrodes (Rossini et al., 2010; Badia et al., 2011, 2016; Lotti et al., 2017).

The chronically implanted devices stimulate the aforementioned multistep cascade of foreign body response, ending in scar tissue formation and electrode encapsulation, and thus in the need of increased currents (i.e., power consumption) to maintain appropriate nerve stimulation due to a progressive increase of the electrical impedance. The most frequently used metals for the fabrication of neural electrodes are gold, tungsten, platinum (Pt) and Platinum-Iridium (Pt-Ir) alloy, with Pt being considered the preferred choice for long-term neuroprosthetic applications due to its electrochemical stability, safety, resistance to corrosion and limited reactivity within a tissue environment (Brummer et al., 1983; Geddes and Roeder, 2003; Merrill et al., 2005; Polikov et al., 2005). However, the stiffness of Pt has a traumatic impact on the surrounding soft neural tissue (Green et al., 2012), causing a shear stress that over time induces an inflammatory reaction, which can be further stimulated by the tissue movements and electrode micromotion (Rousche et al., 2001; Leach et al., 2010). In addition, another weakness of Pt and other metallic electrodes is due to their fabrication, which is usually performed with smooth surfaces that do not allow complete nervous tissue adhesion and integration. As a result, immune cells may invade the remaining space between device surface and target nerve in the implanted area, fostering the FBR (Aregueta-Robles et al., 2014). Therefore, the strength of the implant-tissue integration is influenced by the presence of FBGCs and monocytes/macrophages (Fink et al., 2008). On the other hand, manufacturing excessively rough surfaces may risk increasing the local strain and producing friction forces, thereby causing tissue damage. It is also known that rougher surfaces are able to alter cell adhesion, growth, activation and behavior (Fink et al., 2008; Gamboa et al., 2013; Hulander et al., 2013) including macrophage fusion (Chen et al., 2010), although these effects depend on the different cell types as well as on the materials used and their fabrication methods. Consequently, the right compromise should be sought between the optimal flatness, smoothness and suitable roughness that meet the texture of the nerve tissue, thus avoiding local insults and hazardous damages that could trigger inflammation and a deranged wound healing process. Because of these intrinsic limitations in metallic electrode efficiency, the continuous search for valid alternatives and chemical modifications to material 
composition is encouraged. For example, electrodes can be coated with conductive and soft polymers, like a core of flexible and insulating polyimide with metallic tracks of Pt or Pt-Ir, as detailed below. Such a strategy can be adopted for mitigating the stiffness disparity between device and host tissue and for relieving the biological rejection of the nerve tissue (Geddes and Roeder, 2003; Merrill et al., 2005; Polikov et al., 2005).

So far, diverse strategies are being pursued (Figure 2) to create minimally invasive neural implants that may address the FBR issue and guarantee their long-term use, which can be summarized as follows: (i) working on the design and geometry of the device (such as surface roughness, electrode shape, size, and flexibility); (ii) working on the chemical composition of the coating material to develop novel organic and synthetic polymer substrates that can be tolerated much better by the host tissue. Finally, another important approach consists in (iii) working on the interaction between interface and microenvironment for controlling the local delivery of therapeutic molecules (e.g., anti-inflammatory and anti-fibrotic drugs) making use of functionalized biomimetic and biodegradable coatings.

\section{Working on the Design and Geometry of the Electrode}

The shape and topography of medical-grade polymers implanted in animal models profoundly influences the FBR at the implant surface, with the broadly accepted experimental outcome that circular and smooth surfaces, in intramuscular and percutaneous implants, minimally affect the aggressive behavior of macrophages (Matlaga et al., 1976; Salthouse, 1984). The use of flexible implants of multifunctional polymeric fibers (Canales et al., 2015), and microfabrication of the electrode shape with a new flexible sinusoidal design and a 3D spheroid tip that reduce local strain and tissue damage caused by micromotion (Sohal et al., 2014) may represent alternative strategies to gain some mechanical benefits, without remarkably modifying the size of neural implants, and improve their in vivo longevity and recording performances. The importance to focus on the coating stiffness and geometric configuration (i.e., size effect), to reduce the mechanical mismatch between chronic implanted electrodes and neural tissue, has been highlighted by a recent work of Spencer et al. (2017). They investigated the ability of soft polyethylene glycol dimethacrylate (PEG-DMA) hydrogel coatings, compared to hard implants of identical diameter, to reduce chronic glial scar formation on the surface of neural probes in rodent brains, by lowering the local strain and diameter (from 400 to $150 \mu \mathrm{m}$ ) of the coating. The authors suggest that a similar technique could be adapted to coat more complex geometries through a dip coating, or spray coating method, including electrodes made of various materials, such as metal, silicone and polymer implants, by slightly changing the chemistry.

The strategy of coating neural electrodes with hydrogels of PEG and PEG-based copolymers, leveraging their high versatility, low-fouling and bioinert properties, has long been used with moderate success in many studies (as reported with various examples in the subsection "other advanced biomedical materials") (Wichterle and Lím, 1960; Rao et al., 2011; Gutowski et al., 2015; Heo et al., 2016), although some limitations that were somehow addressed combining PEG with other polymers. However, PEG shows high susceptibility to oxidative damage in vivo, it may activate severe immune response, and its functionalization is usually troublesome, thereby limiting its application for neural interfaces that require long-term stability (Ostuni et al., 2001; Ward et al., 2002; Knop et al., 2010). Likewise, poly(2-hydroxyethyl methacrylate) (PHEMA), which is, together with PEG, the most widely used coating material for implantable devices (Campioni et al., 1998; Ratner, 2002) is susceptible to non-specific protein adsorption, and eventually to fibrotic encapsulation (Zhang et al., 2017), thereby raising the same problems faced with PEG for the extended stability over time and for restraining an immune reaction. Instead, hydrogels made of zwitterionic polymers, such as poly(carboxybetaines), poly(sulfobetaines), and poly(phosphobetaines) (Chen et al., 2005; Jiang and Cao, 2010; Sin et al., 2014a,b) are biocompatible and highly hydrated materials, showing anti-inflammatory and ultralow-fouling characteristics in vivo, which hold great potential to reduce FBR a way better than PEG hydrogels (Jiang and Cao, 2010; Zhang et al., 2013; Wu et al., 2018), as further discussed below in the hydrogel section.

In the last decades, many endeavors have been made in different biomedical and clinical frameworks, merging microengineering and material chemistry skills with molecular biology knowledge, to modify the physicochemical features of implanted interfaces and tuning their structural and surface features with the aim to control the FBR and increase their neurocompatibility. For example, it was initially proposed the use of the focused ion beam technology as high precision machining technique to create and modify the surface morphology of the interface material, up to nanometric scale, by controlling the ion milling of the substrate or its coating in three dimensions, and thereby modulating in vitro the neural cell adhesion (Raffa et al., 2007). Afterward, other promising solutions developed for patterning the design and morphology of the surface are briefly summarized as follows: the generation of combinatorial libraries of cationic polymer coatings in mice (Ma et al., 2011); the intramuscular implantation, in rat spinotrapezius muscles, of biodegradable poly(l-lactide-co-d/llactide) (PLA), as membranes and uncoated electro-spun fiber meshes with a positively charged plasma-polymer coating, to alter material morphology (Lucke et al., 2018). Finally, the development of a method to control surface porosity of poly(2hydroxyethyl methacrylate-co-methacrylic acid) (pHEMA-coMAA) hydrogels, consisting in the fabrication of parallel channels interconnected to a micrometer-sized spherical pore network (Madden et al., 2010). These surface-modified scaffolds were able to increase neovascularization and reduce the inflammation and tissue scarring. This last work represents another smart approach to control channel size and spacing of a functionalizable surface, which can be achieved by varying the dimensions of the microsphere templates. With regard to changing the geometry of the electrode material, the anionic polysaccharide, alginate, is a naturally-derived polymer able to form biocompatible hydrogels, with the addition of divalent cations, to encapsulate 


\section{Coating Strategies}

\section{ii) Chemical Composition of the Coating}

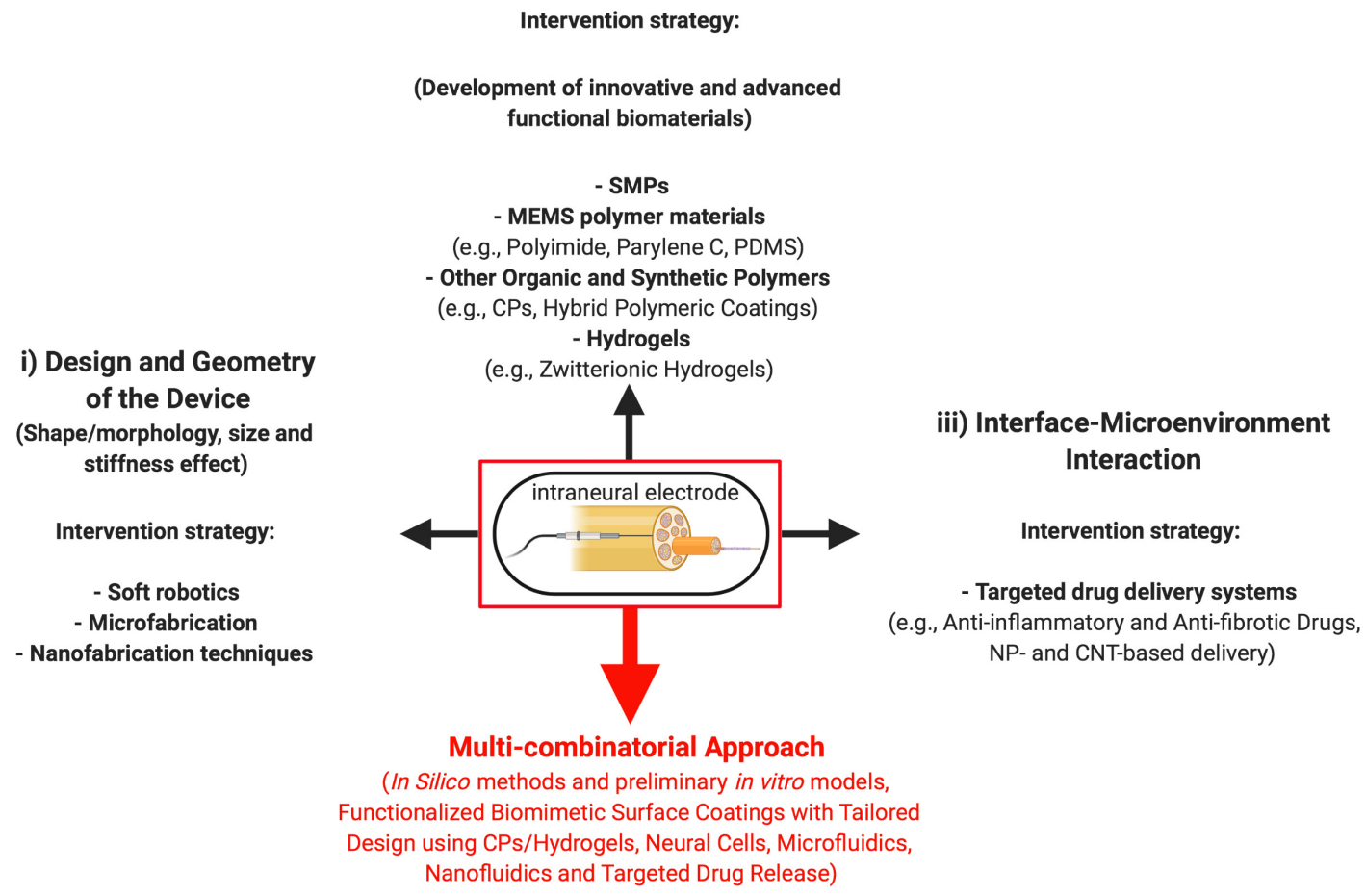

FIGURE 2 | Possible coating strategies of invasive neural implants to minimize the long-term FBR. Schematic representation of the main tissue engineering strategies for coating intraneural electrodes against the FBR: (i) the long-term stability and performance of invasive interfaces can be enhanced through the manipulation of the electrode shape, size, geometry, flexibility and surface roughness to create minimally invasive neural implants by leveraging on micro- and nano-manufacturing methods. (ii) An alternative intervention strategy consists in the development of novel chemical coatings, making use of advanced functional biomaterials, as biocompatible surfaces. (iii) Lastly, the functionalization of the coating with therapeutic drugs and, accordingly, innovative drug-delivery systems may help better integrate and tolerate the invasive device by the host nervous tissue. All of the above intervention strategies could be hopefully integrated into a unique multi-combinatorial approach (red arrow) in the next future. SMPs, shape memory polymers; MEMS, micro-electro-mechanical systems; PDMS, poly(dimethylsiloxane); CPs, conductive polymers; NP, nanoparticle; CNT, carbon nanotube. Created with BioRender.com.

cells and materials for biomedical applications (Lee and Mooney, 2012; Veiseh et al., 2015; Vegas et al., 2016; Bochenek et al., 2018). Semi-permeable alginate spheres have been developed since long time as a common tissue engineering strategy to isolate implanted biological material from the effect of the local immune cells, thus reducing the FBR in vivo (Chang, 1964; Lim and Sun, 1980; Veiseh et al., 2015). Significantly, in one of these works, the authors showed for the first time the importance of the size and spherical geometry not only for the SLG20 alginate hydrogel encapsulation of pancreatic islets, but also for stainless steel, glass and polystyrene spheres on the fibrotic response in immunocompetent and fibrosisprone rodent and non-human primate models (Veiseh et al., 2015). They tested different sizes and time windows, including a chronic time-point (i.e., 6 months), for transplanted grafts encapsulated with the SLG20 alginate capsules and found the $1.5 \mathrm{~mm}$-sized spheres as the ideal geometry to protect grafted cells and surfaces from macrophage activation and fibrosis compared to smaller spheres. In conclusion, they demonstrated that size (1.5 $\mathrm{mm}$ in diameter or greater) and spherical shape, rather than stiffness, of alginate hydrogels as well as ceramic, metal and plastic surfaces represent critical features for obtaining prolonged biocompatibility and for resisting to fibrosis rejection. So, this biomaterial design strategy is potentially applicable to intraneural interfaces although, at present, such dimensions are not always achievable for all the intraneural electrodes. Instead, the strategy proposed by Rubehn and Stieglitz (2010) consists in a novel 3D design of a spiked ultraflexible neural (SUN) interface that integrates spiked structures for intrafascicular nerve recording from the PNS with an ultraflexible substrate, thereby enabling a unique conformal interface to the target nerve. The advantage is represented by the features of the material used, which is an insulating polyimide substrate that does not cause excessive inflammation. Hitherto, this new sensor model has been used only in acute animal experiments, whereas for chronic implantations important challenges still remain to be 
faced such as, among others, the FBR with fibrotic scar tissue that could displace the electrode from its original position and thus jeopardize the quality of the neural signal (Rubehn and Stieglitz, 2010; Wang J. et al., 2018).

Overall, it seems very important to modify the electrode surface with more smooth and circular shape, without major changes in size, to reduce both the local strain of the material and the mechanical mismatch between the device and the host tissue. The consequences of altering the surface topography, in particular the effect of the roughness, are still debated and quite complex to understand (Fink et al., 2008). In fact, topography-induced changes seem to affect macrophage behavior (e.g., cell adhesion, fusion and cytokine secretion in vitro) in the FBR to diverse polymer surfaces (Chen et al., 2010). Furthermore, the continuous search for novel 3D surface design of the device, with coatings at high flexibility, which can be able to adapt to the microenvironment, shaping themselves to the nervous tissue would represent a plus for improving more and more the implant integration. To this aim, 3D bioprinting of hydrogels and thin-film deposition technologies of biocompatible and soft polymers will facilitate the task.

\section{MODIFICATIONS OF THE INTRANEURAL ELECTRODES BY INTEGRATING SOFT ROBOTICS, MICROFABRICATION OF MICROFLUIDIC SYSTEMS AND CARBON NANOTUBES}

In the research field of neural electrodes and probes continuous efforts are being made in search of smaller and more flexible devices to reduce the trauma caused by their insertion and, in turn, the biological tissue response (chronic inflammation and fibrosis), leveraging on micro- and nano-fabrication techniques. Recently, an innovative soft robotics approach has been devised to mitigate the FBR by controlling fluid flow and shear stress perceived by the host cells (Dolan, 2019). In a rat model, the authors implanted subcutaneously a milliscale dynamic soft reservoir (DSR), surrounded by an actuatable polyurethane membrane, and modulated the biomechanics of the biotic-abiotic interface via tunable pressure. After 14 days, an important reduction in the number of $\alpha \mathrm{SMA}+$ myofibroblasts and in fibrotic encapsulation of the implantable device was observed through histological and immunohistochemical analysis. Furthermore, as an example of a proof-of-concept study using a porous and permeable actuating membrane, they were also able to regulate therapeutic delivery of epinephrine, used as a model pharmacological agent, to test its functional effect in the adjacent tissue. Hence, the presented DSR may have the potential to be integrated into intraneural electrodes for an extended period to modulate the inflammatory and fibrotic response, making it a promising tool also for future neural applications. In fact, the design of the platform can be easily modified and tailored to be integrated into diverse types of implantable devices through its incorporation into a thin matrix that can be part of an intraneural electrode. In the past decades, flexible polymer-based microelectrodes have been developed also for neural prosthetic devices (e.g., testing different device size, shape, surface smoothness and structural stiffness) taking advantage of microfluidic and micromachining techniques (Szarowski et al., 2003; Lee et al., 2004; Polikov et al., 2005). Despite these microelectrodes provide multiple and high-quality stimulation and recording sites, the lack of long-term stability has been reported due to the neural tissue reaction and scar formation following extended microelectrodes implantation (Lee et al., 2004). To overcome this limitation, scientists sought to integrate microfluidic channels into flexible microelectrodes combining different techniques for achieving controlled delivery of anti-inflammatory and anti-fibrotic drugs through the microchannels, as further reviewed in Section "InterfaceMicroenvironment Interaction." However, micromachining of the electrode polymer through a lamination technique (Metz et al., 2004), micromolding and thermal bonding of the polymer (Ziegler et al., 2006), combined electrochemical deposition of conductive polymer and drugs on the electrode (Wadhwa et al., 2006), turned out to be complex and expensive for a large-scale use. Hence, novel microelectrodes, combining thinfilm fabrication with poly(dimethylsiloxane) (PDMS) molding and a more rapid, easy, and cost-effective bonding technique, enabled long-term drug release for a more stable recording performance (Gao et al., 2013). A new hybrid cuff electrode that integrates microelectrodes, for recording and stimulation, embedded within microfluidic channels for drug delivery is an example of flexible thin-film polymer device fabricated via surface micromachining techniques on a temporary silicon wafer carrier (Elyahoodayan et al., 2020). The electrode was designed and developed to improve fascicular selectivity and sensitivity in rat sciatic nerves following minimal handling during surgical implantation. Its main advantage is represented by the combined possibility to acutely stimulate, record and deliver lysing drugs, to remove connective tissue (i.e., epineurium layer) that separates electrodes from nerve fibers, and neurotrophic factors that promote axonal sprouting from the exposed fibers. Nevertheless, the authors stated that future studies will be necessary for functional testing in prolonged implant conditions to check for chronic electrophysiological recording as well as nerve health and interface stability after collagenase delivery to verify possible levels of axonal inflammation and fibrosis. Regarding novel and advanced production methods of microelectrodes, a great deal of interest has recently emerged in the additive manufacturing techniques, a versatile and powerful tool to overcome various shortcomings of conventional lithography techniques. Additive manufacturing of microelectrode arrays or microneedle arrays provides a novel, quick and low-cost method to fabricate custom-shaped electrochemical devices, by rapid prototyping, for a wide range of applications (Yang et al., 2016; Morrison et al., 2019; Soltanzadeh et al., 2020). For example, the manufacturing method performed by an aerosol jet technology, for the fabrication of the microelectrode arrays used in a biosensor platform for electrochemical measurements, was based on the use of a silver nanoparticle (NP) ink and a UV-curable polymer (Yang et al., 2016). Instead, in another 
work, compared to microfabricated microneedle arrays, 3Dprinted arrays, made of an amorphous polymer of acrylonitrilebutadiene-styrene, showed almost identical geometric properties and equivalent performance with high frequency biosignals (such as in electromyogram recordings), whereas for recording low frequency signals they turned out to be not suitable (Soltanzadeh et al., 2020). However, in these works, only preliminary and short-term tests were run to measure their functionality (e.g., electrical stimulation in mouse brain, signal recording ability and impedance characteristics) either in human subjects in a noninvasive manner (Soltanzadeh et al., 2020), or in mice (Morrison et al., 2019) and as electrochemical laboratory biosensors (Yang et al., 2016), thus requiring further and deeper in vivo investigation to establish the real advantages and drawbacks of 3D-printed microelectrodes and the biocompatibility of the materials used before their clinical application.

To date, microelectrode technologies present important limitations mainly due to the stiffness mismatch between metals or micromachined silicon, used for electrode microfabrication, and surrounding tissue, particularly soft brain tissue (Winslow and Tresco, 2010). Thus, the mismatch results in fibrotic encapsulation of the microelectrode in chronic implants (Polikov et al., 2005). Furthermore, the problem of controlling possible micromotion of the interface that can change its position in the tissue may also gradually increase the inflammatory reaction (Gilletti and Muthuswamy, 2006). Similar issues can occur with chronic implants of microfabricated peripheral nerve devices. Thus, another group developed a novel fluidic microdrive technology to implant and microactuate ultraflexible electrodes, with a parylene-coated core of carbon nanotube (CNT) fibers, in animal models that could find useful applications also in peripheral nerves (Vitale et al., 2018). Indeed, following fluidic implantation into the nervous tissue, the authors were able to perform electrophysiological recordings, enhancing the stability of the device without the need of increasing the stiffness and thickness of the microdevices, and thus preventing also the onset of inflammatory responses. Fluidic microdrives were fabricated in PDMS by conventional replica molding technique and the microelectrodes insertion was obtained via viscous drag force due to the finely controlled liquid flow in the microfluidic channel, limiting tissue damage at a negligible extent. Such brilliant strategy could be further implemented for peripheral nerve electrodes, envisioning exciting opportunities for their chronic implants. Wireless and flexible film-based ion-selective electrodes (ISEs) have also been recently developed as miniaturized systems for performing highly sensitive and non-invasive measurements (Lim et al., 2020). These sensor systems, made of carbon-polymer composite transducers integrated onto a flexible circuit, enable ions detection in body fluids with high accuracy and selectivity and for prolonged lifetime, showing great potential for their application also in health studies and clinical systems. Another recent approach to drastically reduce the risk of alteration of the performance of the transducer material used for sensors and electrodes, was the development and characterization of solid contact ion-selective electrodes using novel composite material (Kałuża et al., 2019). The formulation of the present nanocomposite was based on multi-walled carbon nanotubes
(MWCNTs) and poly(3-octylthiophene-2,5-diyl) (POT), with the immobilization of the polymer on the carbon nanostructures, preventing its spontaneous and unwanted partition to the membrane phase. The obtained sensors were characterized with good performance, high conductivity as well as high stability of potential readings over time. Nevertheless, although the remarkable electrical and physical properties of CNTs that can be exploited for enhancing the functionality of metallic electrodes (Aregueta-Robles et al., 2014), the main concern for their longterm use in vivo remains related to their cytotoxicity and to the risk of causing intracellular damages. Indeed, because of their elevated stiffness and reduced size (Krishnan et al., 1998), CNTs can easily penetrate cellular membranes (Kagan et al., 2006; Gilmour et al., 2013) and damage nuclei and cytoplasmic organelles. Additionally, they are known to be cytotoxic at high concentrations in different cell types (Bottini et al., 2006; Tian et al., 2006). In spite of such significant risks, which need to be carefully evaluated before clinical applications, nanoscale features of CNTs enable their escape from the immune system surveillance, thereby providing an undoubtedly appealing resource for the future development of innovative intraneural electrodes. A summary of the intervention strategies based on the design and geometry of the electrode with representative examples is reported in Table $\mathbf{1 .}$

\section{Developing Innovative and Advanced Functional Biomaterials}

Recently, other research groups worked on the development of more suitable materials that can be tolerated by the neural tissue, leveraging on material chemistry, micro- and nano-fabrication techniques (Fekete and Pongrácz, 2017). Many different polymers turned out to be possible substrates of neural interfaces due to their proper flexibility, stability, insulation properties and biocompatibility (Svennersten et al., 2011; Ordonez, 2012; Ware et al., 2013; Nguyen et al., 2014; Arreaga-Salas et al., 2015; Boddupalli et al., 2016). Noteworthy, among these are: shape memory polymers (SMPs) [such as polyurethanes, polylactides, polystyrenes, poly(cyclooctene), thiol-enes and poly(vinyl acetate)]; the widely used micro-electro-mechanical systems (MEMS) polymer materials, namely polyimide, parylene C, PDMS and SU-8 (an epoxy-based photoresist suitable for microelectronic applications). In the soft neural tissue, the use of new smart SMPs is gradually overcoming the one of more stiff materials, as the former seem to drastically reduce the inflammatory response in the surrounding tissue becoming compliant after implantation (Ware et al., 2013; Nguyen et al., 2014; Minev et al., 2015). Likewise, in the PNS the use of flexible polymer materials seems to eliminate the mechanical mismatch of compliance between the implanted electrode and the biological tissue (Blakney et al., 2012; Nguyen et al., 2014).

\section{MEMS POLYMER MATERIALS}

\section{Polyimide}

It is a highly resistant and biocompatible polymer, made by imide monomers, among the most widely used substrates 
TABLE 1 | Intervention strategies based on the design and geometry of the electrode.

\section{(i) Design and geometry}

\begin{tabular}{|c|c|c|}
\hline Features & Examples & References \\
\hline \multirow[t]{3}{*}{ Size effect } & PEG-DMA hydrogel coatings and deep and spray coating method & Spencer et al., 2017 \\
\hline & PEG-based coatings & $\begin{array}{l}\text { Reviewed in Knop et al. (2010) } \\
\text { Wichterle and Lím, 1960; Rao et al., 2011; Gutowski } \\
\text { et al., 2015; Heo et al., 2016; Lee et al., } 2017\end{array}$ \\
\hline & PHEMA-based coatings & $\begin{array}{l}\text { Reviewed in Ratner (2002) } \\
\text { Campioni et al., 1998; Jhaveri et al., 2009; Zhang et al., } \\
2017\end{array}$ \\
\hline Shape & Flexible implants of multifunctional polymeric fibers & Canales et al., 2015 \\
\hline \multirow[t]{2}{*}{ Design and topography } & $\begin{array}{l}\text { Physical properties, surface micro-/nano-topography and surface chemistry } \\
\text { modifications }\end{array}$ & $\begin{array}{l}\text { Reviewed in Ware et al. (2013) } \\
\text { Anderson et al., 1999; Thull, 2002; Fink et al., 2008; } \\
\text { Chen et al., 2010; Gamboa et al., 2013; Hulander et al., } \\
2013\end{array}$ \\
\hline & 3D design of spiked ultraflexible substrates & Rubehn and Stieglitz, 2010; Wang M. et al., 2018 \\
\hline \multirow[t]{2}{*}{ Surface porosity } & Channel size control through (pHEMA-co-MAA) hydrogels & Madden et al., 2010 \\
\hline & PU-based porous implants & Ward et al., 2002 \\
\hline Size and spherical geometry & Alginate spheres/capsules & Veiseh et al., 2015 \\
\hline \multicolumn{3}{|l|}{ Intervention strategy } \\
\hline Soft robotics & $\begin{array}{l}\text { Control over fluid flow and shear stress through milliscale dynamic soft reservoir } \\
\text { with actuatable membrane }\end{array}$ & Dolan, 2019 \\
\hline \multirow[t]{2}{*}{ Microfabrication } & $\begin{array}{l}\text { Micro-machined neural prosthetic devices: flexible polymer-based } \\
\text { microelectrodes with different shape, size and geometry }\end{array}$ & $\begin{array}{l}\text { Reviewed in Szarowski et al. (2003); Lee et al. (2004), } \\
\text { Metz et al. (2004); Polikov et al. (2005), Spataro et al. } \\
\text { (2005); Ziegler et al. (2006), Winslow and Tresco (2010); } \\
\text { Blau et al. (2011), Gerwig et al. (2012); Gao et al. } \\
\text { (2013), Minev et al. (2015); Qi et al. (2017), Vitale et al. } \\
\text { (2018); Kozai (2018), Fallahi et al. (2019), and Kumar } \\
\text { et al. (2020) } \\
\text { Elyahoodayan et al., } 2020\end{array}$ \\
\hline & Additive manufacturing of microelectrode arrays and microneedle arrays & $\begin{array}{l}\text { Yang et al., 2016; Morrison et al., 2019; Soltanzadeh } \\
\text { et al., } 2020\end{array}$ \\
\hline \multirow[t]{11}{*}{ Nanofabrication } & CNTS & $\begin{array}{l}\text { Reviewed in Aregueta-Robles et al. (2014) } \\
\text { Castagnola et al., } 2016\end{array}$ \\
\hline & Parylene-coated flexible CNTf microelectrodes & Vitale et al., 2018 \\
\hline & Conducting-polymer carbon nanotubes & $\begin{array}{l}\text { Abidian et al., 2010; Gerwig et al., 2012; Alba et al., } \\
\text { 2015; Mandal et al., 2015; Samba et al., 2015; Du } \\
\text { et al., 2018; Altun et al., 2019; Kałuża et al., 2019; } \\
\text { Zheng et al., } 2019\end{array}$ \\
\hline & PPy nanowires & Reviewed in Qi et al. (2017) \\
\hline & PPy nanoparticles & Hosseini-Nassab et al., 2017 \\
\hline & SWCNT-PPy/PEGDA composite hydrogels & Xiao et al., 2012 \\
\hline & PPy/CNT films & Luo et al., 2011 \\
\hline & Graphene oxide nanocomposite films of PPy & Weaver et al., 2014 \\
\hline & PLGA nanoparticles embedded in alginate hydrogels & Kim and Martin, 2006 \\
\hline & Nanoparticle-coated nanoelectrodes & Bazard et al., 2017 \\
\hline & Nanoscale biomimetic surfaces & Reviewed in Von Der Mark et al. (2010) \\
\hline
\end{tabular}

PEG, polyethylene glycol; DMA, dimethacrylate; PHEMA, poly(2-hydroxyethyl methacrylate); FIB, focused ion beam; PLA, poly(l-lactide-co-d/I-lactide); pHEMA-co-MAA, poly(2-hydroxyethyl methacrylate-co-methacrylic acid); PU, polyurethane; CNTS, carbon nanotubes; CNTf, carbon nanotube fiber; PPy, polypyrrole; SWCNT-PPy/PEGDA, single-walled carbon nanotubes-polypyrrole/poly(ethylene glycol) diacrylate; PLGA, poly(lactic-co-glycolic acid). References: except were specifically indicated as 'Reviewed in,' all others are research articles. 
for the fabrication of the core of novel neural electrodes with metallic tracks, such as $\mathrm{Pt}$ and gold, often coated with different biomaterials for counteracting and delaying the onset of the FBR (Oddo et al., 2016; Delgado-Martínez et al., 2017; Wurth et al., 2017; de la Oliva et al., 2018c). Indeed, among the possible neuroprosthetic applications of this polymer, the group of Navarro X. developed a novel double-aisle electrode to regenerate separated nerve fascicles, made of a double-side thin-film of polyimide (Delgado-Martínez et al., 2017). Although such interface allowed regeneration of nerve branches, it caused FBR in chronic implants. The reaction was indeed similar to that obtained previously with other chronically implanted polyimide intrafascicular electrodes and non-obstructive regenerative electrodes (Lago et al., 2007; Garde et al., 2009), thus affecting the quality of neural signal over time. This common limitation when using polyimide electrodes might be overcome through the functionalization of the polyimide core with advanced biomimicry ultra-low fouling organic or synthetic coatings that can be much more tolerated by the implanted tissue. Toward this direction, diverse efforts have been made to reduce the inflammatory response and electrode encapsulation through new biomimetic solutions. One of these involved the coating with bioresorbable layers of molten saccharose for intracortical insertion in rat models (Hassler et al., 2016). Another option was a superhydrophobic coating from a natural Xanthosoma sagittifolium leaf nanocasted on an electroactive polyimide surface (Chang et al., 2013). A different nanotechnological approach was attempted using hybrid conductive material: an indium tin oxide substrate associated to a nanostructured polyimide film deposited on a glass surface, using a new and simple nanopatterning technique (Rombaut et al., 2019). Very recently, a flexible and transparent polyimide-based electrode was fabricated with a trilayer-stacked geometry that exploits the properties of a high-quality ultrathin film of graphene. This solution showed enhanced power and current efficiencies, with properties comparable to indium tin oxide-based diodes, increased flexibility and long-term stability in different devices (Lee et al., 2019). Finally, another strategy to increase the longterm reliability, while maintaining high flexibility, of a polyimidebased neural interface in free-moving rats, was the one adopted by a research group from China, through a MEMS fabrication approach (Ji et al., 2018). This group developed an innovative optogenetics tool consisting in a polyimide-based hybrid (optoelectric) flexible device that integrates 16 micro-LEDs and 16 IrOx-modified microelectrode arrays. Such device allowed simultaneous, high-resolution optical stimulation and electrical recording of cortical areas. Using this tool, they observed little reduction in the electrical or optical performance for 3 months. Although the fabrication process was quite complex, the device revealed itself to be a promising neural interface for further neuroscience applications, expandable also to larger animals (e.g., non-human primates) and possibly to human patients. However, in order to evade the issue of non-specific protein and cell absorption on the polyimide surface, several groups tried to devise valid alternatives to polyimide substrates, using either diverse MEMS polymers or newly emerged biomedical materials, as shown below.

\section{Parylene C}

Parylene $\mathrm{C}$ is a variety of high flexible and chemically inert poly(p-xylylene) polymer commonly used as biocompatible coating and substrate material of electrodes for soft neural implants (Fekete and Pongrácz, 2017). In a recent work, the authors tested parylene $\mathrm{C}$ as a substrate material for peripheral nerve interfaces both in vitro and in vivo (de la Oliva et al., 2018a). In this study, longitudinal devices made of parylene $\mathrm{C}$ and polyimide were implanted in the rat sciatic nerve for up to 8 months and the induced FBRs were compared one another. In spite of the advantage to produce parylene C-based thinner substrates than polyimide ones, with no harmful effect on nerve function, long-term stability of such electrodes could be affected by a thicker tissue capsule than polyimide devices. Indeed, the authors observed much more fibroblasts surrounding the former device, thus making parylene $\mathrm{C}$ not suitable for chronic implantations (Lecomte et al., 2017; Mueller et al., 2017; de la Oliva et al., 2018a). However, the diverse pattern of FBR around parylene $\mathrm{C}$ vs. polyimide, due to their different chemical structures, deserves further investigation before parylene $\mathrm{C}$ drops out of other possible invasive neural applications. For example, in another study the authors microfabricated and tested in vivo up to 24 months, even though in the rabbit brain, a sinusoidal probe electrode made of a tungsten titanium alloy (WTi) core encased in flexible layers of parylene $\mathrm{C}$ with novel design features (Sohal et al., 2014). Interestingly, over the chronic experimental period of the study the electrode performances and neuronal integration were better than other conventional electrodes used for recording of neuronal activity in humans, showing low levels of gliosis. Another interesting attempt to improve the long-term stability in vivo of an intrafascicular neural interface (i.e., a flexible microelectrode array with a recording system), was made through a mechanically enhanced flexible interconnection cable using a combination of parylene C and polyimide (Kang et al., 2019). The former provided chemical and electrochemical stability while the latter improved the mechanical strength and handling, with no damage reported, during the implantation procedure of the whole neural interfacing device in canine sciatic nerves. However, before clinical translation, these promising results need more investigation to test their reproducibility in chronic implants of peripheral nerves in larger animal models. Despite the many benefits of parylene $\mathrm{C}$ as conformational coating, such as its chemical inertness, there are also significant disadvantages that can limit its wider application compared to the liquid epoxy or silicon coatings. Notably, a better performance and a more controlled deposition process of the latter that are, moreover, much more cost-effective in their production-run make them a preferable choice for researchers. Furthermore, the chemical vapor deposition process required to apply parylene $\mathrm{C}$ onto a surface, especially a conductive-metal one, is not only timeconsuming but also costly in the attempt to increase its metal adhesion through different methods.

\section{Poly(Dimethylsiloxane) (PDMS)}

This silicon-based organic polymer is the elective material for microfabrication of microfluidic devices including 
microelectrodes, with tissue-like elastic modulus, easily compliant to neural tissue. These flexible electrodes are usually realized through the process of replica molding, from a master obtained by soft photolithography with a SU-8 photoresist (Qin et al., 2010). Alternatively, they can be fabricated via simple and cost-effective photolithography-free methods, such as laser micromachining and master molding of PDMS. Such versatile processes give rise to planar metal electrodes with microfluidic channel geometries (Chatzimichail et al., 2018), and stable neural interfaces (Gao et al., 2013; Minev et al., 2015).

Poly(dimethylsiloxane) micromachining is not only cheap, and easy to realize with high parallelization, but also suitable for the fabrication of long-term neural implants that are able to produce lower inflammatory response than polyimide-based electrodes (Minev et al., 2015). Flexibility and elasticity of PDMS are clearly advantageous features for the fabrication of neural electrodes, as well as in promoting neuronal maturation (Teixeira et al., 2009; Yang and Suo, 2018). Notwithstanding, because of PDMS hydrophobicity, achieving its stable adhesion to hydrated surfaces and materials, such as hydrogels, can be problematic (Yang and Suo, 2018). Furthermore, the proper stability and adhesion between different layers of elastic polymers in implantable electronic devices, such as stretchable electrodes, is difficult to achieve. Actually, under the pressure of muscle contraction and of the strain imposed by the micromotion between nerve tissue and the implant, the electrode can crack. This issue can eventually jeopardize the device functionality. Therefore, alternative solutions have been pursued using allpolymer and metal-free microelectrode arrays with a mixture of various stretchable polymers and via replica molding with PDMS (Blau et al., 2011; Guo et al., 2014; Qi et al., 2017), although with mixed fortunes, as described in the next section.

\section{OTHER ADVANCED BIOMEDICAL MATERIALS}

From the close collaboration between the bioengineering field and the biomedical research area in the development of novel biomaterials for chronic neural applications, diverse strategies are being pursued to decrease the FBR in the next-generation neural interfaces. Some of them are based on the use of organic and synthetic polymeric coatings, including conductive polymers (CPs). Among organic coatings, CPs have been recently investigated with the aim to improve the long-term performance of neural electrodes as they can increase their effective surface, thereby decreasing the impedance, and enhance the electrical properties of neural interfaces, thus seeming the most promising materials (Wilks et al., 2011; Charkhkar et al., 2016). In particular, Poly (3,4-ethylenedioxythiophene) PEDOT, and some of its modified and hybrid versions, have been shown to be safe and reliable candidates in neuroprosthetic applications, being stable and able to improve neural adhesion, electrochemical impedance and dramatically reduce electrical noise and host tissue response (Abidian et al., 2010; Green et al., 2013; Ferlauto et al., 2018; Ganji et al., 2018). Moreover, PEDOT can be easily doped and bio-functionalized with anti-inflammatory drugs, such as dexamethasone (Alba et al., 2015; Boehler et al., 2017; Kleber et al., 2019). It can also be conjugated with other biocompatible and bioinert materials, such as PDMS thin films, CNTs, tetrafluoroborate (TFB), poly(styrenesulfonate), alginate and nafion to guarantee electrochemical stability both in vivo and in vitro (Blau et al., 2011; Alba et al., 2015; Charkhkar et al., 2016; Ferlauto et al., 2018; Carli et al., 2019). To date, PEDOT functionality has already been demonstrated in vitro in terms of improvement of neurite outgrowth bioactivity, and stability of neural micro-stimulation (Green et al., 2009; Mandal et al., 2015). Nonetheless, the long-term performance and integrity in vivo of such coatings for chronic recordings have yet to be verified, despite some interesting data collected from short-term epicortical and epidural recordings (Blau et al., 2011). However, these aspects start to be evaluated with promising long-term results, such as for the chronic intracortical neural recordings with high stability and activity in rat motor cortex and mice visual cortex, which deserve further investigation (Charkhkar et al., 2016; Ferlauto et al., 2018; Carli et al., 2019). Another important example was provided by a research team that developed a metal-free electrode array of polypyrrole/polycaprolactoneblock-polytetrahydrofuran-block-polycaprolactone (PCTC) sandwiched in between films of PDMS. This group compared the in vivo performance of such all-polymer interface with a Pt electrode of the same area in a rat (Guo et al., 2014). They demonstrated a lower impedance of the metal-free device, along with excellent electrical stimulation performances in a stimulated rat hind-limb muscle following squeezing of the sciatic nerve and higher charge injection capacity compared to the Pt electrode, as well as to other PEDOT-coated metal electrodes. Future work from the same group will be necessary to improve and characterize the device physical integrity and mechanical performance in long-term in vivo assays also in peripheral nerves.

Two of the most widely used synthetic polymers for coating electrodes are poly (ethylene glycol) PEG (Drury and Mooney, 2003; Gutowski et al., 2015) and PHEMA (Jhaveri et al., 2009; Mario Cheong et al., 2014), as they can form hydrogels with low- or non-fouling characteristics in vivo, thus enhancing tissue response around implanted electrodes. However, their long-term use is limited by oxidative mechanisms that partially compromised non-specific protein absorption and device performance. Therefore, recent hybrid solutions have been proposed to overcome some of the issues related to their prolonged stability and sensitivity in vivo, such as hybrid thin film photopatternable polymers, combining the properties of PEDOT with the long-term (over 10 days) moisture stability of PEG (Zhu et al., 2017). Another successful test was the integration between PEDOT-poly(styrene sulfonate) (PSS)-CNT nanocomposites and biocompatible PHEMA hydrogels (Castagnola et al., 2016), for potential acute and chronic flexible and high sensitivity electronic applications in rat brains. Thus, the PHEMA hydrogel was able to guarantee the electrochemical performance of the device and improve the quality of intracortical recording until 28 days after the implant, along with the advantage of reducing the mechanical mismatch between neural tissue and device preventing the nanomaterial detachment. Instead, other 
researchers produced a polydopamine-based coating, resistant to protein adsorption, also for potential applications in intraneural electrodes (Kwon et al., 2016). They developed a polydopamine melanin (PDM) film in the nanometer-scale, a synthetic analog of the two naturally-occurring chemicals dopamine and eumelanin holding unique ionic and electronic properties (Ambrico et al., 2013, 2014; Wünsche et al., 2013), which could be harnessed to increase neural electrodes performance by improving their in vivo biocompatibility, while reducing their interfacial impedance. However, further studies will be needed to verify the potentiality of such PDM films. Another group biofunctionalized roughened Pt black (BPt) peripheral nerve cuff electrodes for chronic implantation in animal models using two coatings of PEG or nafion, with the latter showing low interfacial impedance, together with good stability and reduced fibrotic capsule, thus justifying deeper investigation also for possible clinical applications (Lee et al., 2017). A different research team developed a novel CP for neural electrodes made of a soft wire conductive matrix, which showed optimal mechanical (suitable flexibility) and electrochemical properties, as well as excellent biocompatibility after 1 month implantation in a rat sciatic nerve (Zheng et al., 2019). The conducting core of the electrode was based on silicone/poly(3,4-ethylenedioxythiophene)polyethylene glycol (PEDOT-PEG) elastomer encapsulating 3D CNTs, and it was shown to be more compliant to soft nerve tissue than traditional polyimide implants in terms of FBR. Finally, another CP frequently used as electromechanically active coating for biosensors, implantable gold electrodes (Yamato et al., 1995; Cui et al., 2003; Green et al., 2008), fiber scaffolds capable of dynamic mechanical actuation (Gelmi et al., 2016) and microelectrode arrays (Qi et al., 2017; Du et al., 2018), is the polypyrrole (PPy). However, it has often shown limited performances and chronic recording failure over extended periods of time in vivo, also due to chronic inflammation and fibrotic encapsulation (Yamato et al., 1995; Cui et al., 2003; McConnell et al., 2009). In a recent work, a research group tried to improve the performance of biosensing interfaces based on copolymerization of benzenamine-2,5-di(thienyl)pyrrole (SNSAn) with 3,4-ethylenedioxythiophene (EDOT) (Altun et al., 2019). The so-developed copolymer films showed increased biosensing efficiency after the incorporation of CNTs and fullerene, albeit evidence of the effect of such copolymerization on their performance in vivo is still missing. Conversely, others observed high conductivity and good performances in their in vivo recordings of rat electrocorticographic signals, and in the stimulation of the sciatic nerve of the animals through the use of stretchable polymeric microelectrode arrays. These arrays were composed of PPy electrodes anchored to an underlying PDMS film using PPy nanowires. Moreover, these flexible devices showed high stretchability with no cracking, high resistance up to $100 \%$ strain and good electrode-substrate adhesion (Qi et al., 2017). To sum up, composite PEDOT-PEG or PEDOTPHEMA solutions would seem to offer a suitable compromise between long-term mechanical and bio-stability as well as high electrical performance ensuring, at the same time, very good biocompatibility, if were not for the current limit of the few available in vivo results against FBR.

\section{HYDROGELS}

The use of highly hydrated and ultralow-fouling polymeric hydrogels outperforms other coating materials in terms of biocompatibility although the existing issue of the low electrical properties of some chemical hydrogel compositions. This drawback could be solved by including in hydrogels some of the conductive components examined above, such as CPs and CNTs (Green et al., 2012; Xiao et al., 2012). Another alternative solution could be the use of zwitterionic hydrogels with ionic conductive capacity as well as biomimetic and antiinflammatory features, which can also resist the FBR for longer time-scale than other synthetic HEMA hydrogels (Zhang et al., 2013; Diao et al., 2019). For instance, in one of these most recent papers, it was demonstrated that highly stretchable, tough and flexible PVA/P(AM-co-SBMA) zwitterionic hydrogels possess high intrinsic ionic conductivity due to the zwitterionic counterions, and could therefore fulfill flexible electrical device applications (Diao et al., 2019). Further examples are represented by the synthesis of ultralow-fouling zwitterionic hydrogels and non-leaching polymeric sulfobetaine (polySB) coatings for subcutaneous implantation of medical devices in animal models up to 2-3 months (Smith et al., 2012; Zhang et al., 2013; Yesilyurt et al., 2017). Another recent paper showed the synthesis and in vitro validation of poly(carboxybetaine) zwitterionic hydrogel coating, with a Young's modulus in the range of the neural tissue, of a polyimide-based device to minimize the fibroblast and macrophage adhesion (Trel'Ová et al., 2019). Similarly, a previous carboxybetaine methacrylate zwitterionic hydrogel synthesized via photopolymerization, rather than thermal polymerization, with a more reactive and functionalizable crosslinker showed superior stability at diverse $\mathrm{pH}$ values and improved mechanical properties than many other photopolymerized hydrogels (Carr et al., 2011). Finally, in their work some researchers developed a well-controllable electrochemically-mediated surface-initiated atom transfer radical polymerization (e-siATRP) method to fabricate a superlow protein absorption zwitterionic hydrogel coating that was based on poly(sulfobetaine methacrylate) (pSBMA) (Hu et al., 2015). The main advantage of the present method is represented by the usage of the commercially available SBMA and its very easy and controllable synthesis process, which can be also applied to implantable neural electrodes with optimal biocompatibility and antifouling capacity as proven by in vitro tests (Hu et al., 2015).

Besides, another frequently encountered issue related to such systems is the delamination of the hydrogel from the electrode surface, and thus the establishment of adequate patterning methods for binding it to the substrate. In a recent work, microsystem engineers and chemists addressed these problems by developing a new hybrid conductive system made from the combination between the synthetic hydrogel P(DMAA-co$5 \% \mathrm{MABP}-\mathrm{co}-2,5 \% \mathrm{SSNa}$ ) and the conducting polymer PEDOT, which can be covalently attached to the electrode surface and patterned using a photolithographic process via UV irradiation. In such a way, the authors created an interpenetrating network, suitable for coating neural microelectrodes, showing excellent electrochemical stability and no toxicity in vitro (Kleber et al., 
2017). Conductive hydrogel coatings can ameliorate the electrical properties and performances of conventional metal electrodes, with lower energy demand to interface with and control target nerve activity. To achieve a suitable response from a distant stimulated nerve, the application of higher currents is necessary with possible adverse reactions, such as the corrosion of the uncoated metallic electrode and its failure over time. Hence, due to their high efficiency and electrochemical stability, conductive hydrogels can provide stable and long-term activity also when applied to stainless steel (SS) electrode arrays in peripheral nerves as showed in this work (Staples et al., 2018). The researchers fabricated planar electrode arrays by electrodepositing a thin layer of PEDOT/pTS onto the SS electrode and then coating it with a $20 \mathrm{wt} \%$ poly(vinylalcohol)-methacrylate-taurine (PVAtaurine) hydrogel. In their in vitro tests the conductive hydrogel coating improved electrochemical properties and device stability over 42 days regardless of the underlying metallic substrate of the electrode. Nonetheless, the authors used non-penetrating cuffelectrodes and only for in vitro analysis, thereby the benefit of such hydrogel coating against FBR over chronic invasive implant periods in vivo will be the focus of their future work. Accordingly, their principal task will be the demonstration of low scar tissue development due to the reduced hydrogel stiffness and to its natural anti-fouling properties.

Modulation of the FBR for intraneural interfaces can also be achieved taking inspiration from recent works in animal models of type-I diabetes (Vegas et al., 2016; Bochenek et al., 2018). In these in vivo studies the authors performed encapsulation of human pancreatic $\beta$-cells with chemically modified alginate formulations [i.e., triazole-thiomorpholine dioxide (TMTD) alginate, Z2-Y12 and Z1-Y15 immunemodulating alginate derivatives] to long-term protect cells from the chronic response of the immune system, without the need for broad immunosuppression. In particular, these different hydrogel formulations increased the immunoprotection of cells in immune competent mice and non-human primate models, successfully reducing FBR and preventing from pericapsular fibrotic overgrowth. Similar strategies with alginate hydrogels could therefore be translated into clinical practice to encapsulate intraneural electrodes, and exploited to overcome the challenge of foreign body rejection from the host immune system. Overall, despite the many advantages provided by conductive hydrogel coatings in terms of high electrochemical performance of the device, especially when using zwitterionic formulations, augmented quality of signal recording, reduction of the mechanical mismatch along with ultralow-fouling properties, their long-term stability and functionality in vivo still represent main limitations that need to be solved in the next future. In fact, because of their soft texture, highly hydrated jelly structure and low mechanical strength, hydrogels can be slowly degraded or damaged already during the implantation surgery, thus impairing their permanence and performance within neural tissue. However, to the best of our knowledge, at present they are by far the most promising biomimetic coatings in this context.

A summary of the intervention strategies based on the development of advanced functional biomaterials with representative examples is reported in Table 2.

\section{Interface-Microenvironment Interaction}

The aqueous characteristic of synthetic and organic hydrogel coatings, such as PEG-based and zwitterionic-based formulations, and their synthesis methods could be harnessed for therapeutic purposes. In order to modulate locally the immune response of the host tissue, various hydrogel formulations could represent a means to encapsulate or covalently incorporate growth factors, therapeutic anti-inflammatory and anti-fibrotic medications as well as small-molecule drugs (Jhaveri et al., 2009; Mario Cheong et al., 2014; Gutowski et al., 2015; Doloff et al., 2017). To this aim, a considerable list of potential therapeutic drugs could be loaded during polymer fabrication into biodegradable CPs, polymeric coatings and hydrogels (Lotti et al., 2017; Zeglio et al., 2019), and many others could be tested as good candidates for contrasting FBR. In the following sections we will take into account some of the most promising lead compounds and novel drug delivery strategies to further improve the biological response to the electrodes in chronically implanted nerve tissues.

\section{DEXAMETHASONE}

One of the most frequently anti-inflammatory agents loaded into electrode coatings for chronic applications is the corticosteroid drug dexamethasone and its phosphate derivative (Spataro et al., 2005; Kim and Martin, 2006; Mercanzini et al., 2010; Alba et al., 2015). Interestingly, in two FBR models in the rat sciatic nerve, one with longitudinal parylene $\mathrm{C}$ intraneural implants, and the other with longitudinal polyimide-based implants, the beneficial effects of dexamethasone were clearly demonstrated (de la Oliva et al., 2018b). In fact, in this work only subcutaneous administration of dexamethasone up to 8 weeks, compared to other anti-inflammatory drugs (i.e., ibuprofen, maraviroc, and clodronate liposomes), was able to reduce the inflammatory reaction as well as matrix deposition around the electrodes in a comparable manner. In another model of FBR, developed by the same group, using TIME interfaces implanted in the rat sciatic nerve, the long-term functionality (i.e., 3 months) of the electrodes was maintained by systemic administration of dexamethasone. The drug was indeed able to reduce the loss of functioning contacts of the TIMEs that stimulated the target nerves and evoked a muscle response while reducing the inflammatory cell infiltration during the first month, which is the critical time-frame for FBR development (de la Oliva et al., 2019). Since dexamethasone showed similar beneficial effects in different devices and substrates, it may represent an ideal drug treatment to extend the implant functionality over time in peripheral nerves. Accordingly, the use of dexamethasone could be combined with tissue engineering strategies, such as substrate functionalization with biodegradable hydrogels and porous CPs, for its controlled local release in order to specifically target its activity around the implant, while reducing potential side effects caused by its systemic toxicity at too high doses. In relation to this approach, one of the first in vitro attempts to control the release of dexamethasone from a conducting polymer coating of PPy on $\mathrm{Au}$ electrode 
TABLE 2 | Intervention strategies based on the development of advanced functional biomaterials.

(ii) Advanced functional biomaterials

\begin{tabular}{|c|c|c|}
\hline Intervention strategy & Examples & References \\
\hline \multirow[t]{4}{*}{$\begin{array}{l}\text { Novel flexible and biocompatible } \\
\text { polymers }\end{array}$} & Extended overview & $\begin{array}{l}\text { Reviewed in Ordonez (2012); Ware et al. (2013), Boddupalli et al. (2016), and } \\
\text { Fekete and Pongrácz (2017) }\end{array}$ \\
\hline & Polypyrrole microactuators & Svennersten et al., 2011 \\
\hline & $\begin{array}{l}\text { Hydrogel core of bacterial cellulose and } \\
\text { conductive polymer shell layer of PEDOT }\end{array}$ & Chen et al., 2017 \\
\hline & PEG-RGD hydrogels & Blakney et al., 2012 \\
\hline \multirow[t]{3}{*}{ SMPs } & Extended overview & Reviewed in Ware et al. (2013) \\
\hline & Bioinspired cellulose nanocomposites & Nguyen et al., 2014 \\
\hline & Thiol-ene based softening substrates & Arreaga-Salas et al., 2015 \\
\hline \multirow[t]{3}{*}{$\begin{array}{l}\text { Micro-electro-mechanical systems } \\
\text { (MEMS) polymer materials }\end{array}$} & Polyimide & $\begin{array}{l}\text { Reviewed in Kozai (2018) } \\
\text { Lago et al., 2007; Garde et al., 2009; Mercanzini et al., 2010; Chang et al., 2013; } \\
\text { Hassler et al., 2016; Oddo et al., 2016; Boehler et al., 2017; Delgado-Martínez } \\
\text { et al., 2017; Wurth et al., 2017; de la Oliva et al., 2018b,c; Ji et al., 2018; Kang } \\
\text { et al., 2019; Lee et al., 2019; Rombaut et al., } 2019\end{array}$ \\
\hline & Parylene C & $\begin{array}{l}\text { Reviewed in Fekete and Pongrácz (2017) } \\
\text { Ziegler et al., 2006; Sohal et al., 2014; Xie et al., 2014; Lecomte et al., 2017; } \\
\text { Mueller et al., 2017; de la Oliva et al., 2018a,b; Vitale et al., 2018; Kang et al., } 2019\end{array}$ \\
\hline & PDMS & $\begin{array}{l}\text { Blau et al., 2011; Gao et al., 2013; Guo et al., 2014; Minev et al., 2015; } \\
\text { Chatzimichail et al., 2018; Kumar et al., } 2020\end{array}$ \\
\hline CP coatings & Extended overview & Reviewed in Aregueta-Robles et al. (2014) and Balint et al. (2014) \\
\hline \multirow[t]{10}{*}{ Hydrogels } & Alginate hydrogels & Reviewed in Lee and Mooney (2012) \\
\hline & PEG-containing hydrogels & Spencer et al., 2017; Zhang et al., 2017 \\
\hline & PEG-maleimide hydrogel coatings & Gutowski et al., 2015 \\
\hline & Poly(SB) hydrogels & Smith et al., 2012 \\
\hline & PEDOT:PSS/alginate conductive hydrogels & Ferlauto et al., 2018 \\
\hline & Conducting PEDOT/PDMAAp hydrogels & Kleber et al., 2017, 2019 \\
\hline & PHEMA hydrogels & Jhaveri et al., 2009; Castagnola et al., 2016; Zhang et al., 2017 \\
\hline & Conducting hydrogels with biomolecules & $\begin{array}{l}\text { Reviewed in Aregueta-Robles et al. (2014) and Lotti et al. (2017) } \\
\text { Green et al., 2012; Mario Cheong et al., 2014; Chen et al., 2017; Staples et al., } \\
2018\end{array}$ \\
\hline & SWNT-PPy/PEGDA composite hydrogels & Xiao et al., 2012 \\
\hline & Chemically-modified alginate microspheres & Vegas et al., 2016; Bochenek et al., 2018 \\
\hline \multirow[t]{6}{*}{ Zwitterionic hydrogels } & Phosphorylcholine polymer & Yesilyurt et al., 2017 \\
\hline & PVA/P(AM-co-SBMA) polyelectrolyte & Diao et al., 2019 \\
\hline & Poly(carboxybetaine) and pCBMA & Jiang and Cao, 2010; Carr et al., 2011; Zhang et al., 2013; Trel’Ová et al., 2019 \\
\hline & $\begin{array}{l}\text { Phosphorylcholine self-assembled } \\
\text { monolayers }\end{array}$ & Chen et al., 2005 \\
\hline & Poly(sulfobetaine) and pSBMA & $\begin{array}{l}\text { Reviewed in Sin et al. (2014a) } \\
\text { Jiang and Cao, 2010; Sin et al., 2014b; Hu et al., 2015; Wu et al., } 2018\end{array}$ \\
\hline & $\begin{array}{l}\text { Zwitterionic hydrogels with bioactive } \\
\text { materials }\end{array}$ & Reviewed in Von Der Mark et al. (2010) \\
\hline
\end{tabular}

SMPS, shape memory polymers; RGD, Arg-Gly-Asp motif; MEMs, micro-electro-mechanical systems; PDMS, poly(dimethylsiloxane); CPs, conductive polymers; PEG, polyethylene glycol; Poly(SB), polymeric sulfobetaine; PEDOT:PSS, poly(3,4-ethylenedioxythiophene):Polystyrene sulfonate; PEDOT/PDMAAp, poly(3,4-

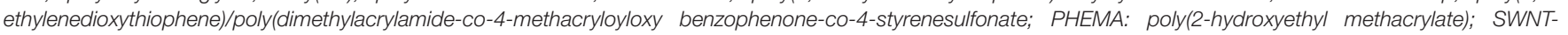

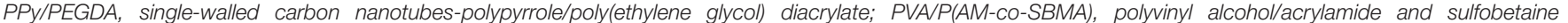

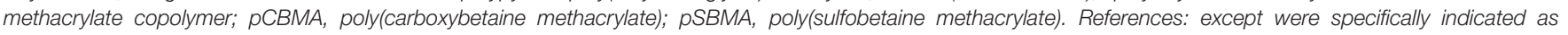
'Reviewed in,' all others are research articles.

sites was done through an electrochemically-controlled release of dexamethasone phosphate as a dopant (Wadhwa et al., 2006). The authors elicited an anti-inflammatory response in murine glial cells, although they experienced a low adhesion of the coating to the electrode, turning out to be unable to sustain an extended drug-delivery time. Instead, MWCNT and dexamethasone-doped electropolymerized PEDOT coatings have shown promise to improve chronic neural electrode performance. Indeed, despite the impedance increase, coated electrodes successfully recorded neural activity throughout the implantation period (Alba et al., 2015), and showed excellent stability and no signs of inflammation, in response to electrical stimulation, over 45 days in rat brain. Similarly, another team filled MWCNTs with a solution of dexamethasone phosphate 
and then sealed the open ends of the nanotubes with a film of $\mathrm{PPy}$, via electropolymerization, as electrode coating for an ondemand drug release strategy (Luo et al., 2011). The researchers detected an effective anti-inflammatory activity in vitro, and the smaller the size of the nanotubes the higher the drug release. Furthermore, such PPy coating significantly decreased the electrode impedance. However, despite some preliminary evidence of the dexamethasone success, there are still a few reliable data in vivo and some considerable kinks to work out before long-term use of the drug as a resolutive antiinflammatory treatment for clinical applications in humans. For instance, an important issue, not only related to dexamethasone but to any other loaded chemicals, is that of the drug exhaustion around the implant microenvironment.

\section{ANTI-FIBROTIC DRUGS}

It has recently been found another molecular target underpinning the development of the FBR. Actually, targeting colony stimulating factor-1 receptor (CSF1R), which is upregulated on the macrophage surface after implantation of different biomaterials, including biocompatible hydrogels, may represent a smart strategy to hamper fibrosis and capsule formation (Doloff et al., 2017). Such therapeutic approach may indeed avoid to directly targeting macrophages or applying massive immunosuppression with possible harmful side effects to the whole organism.

Another potential target protein is the connective tissue growth factor (CTGF), a key player underlying the progression of the fibrotic reaction driven by TGF- $\beta$, which is quickly induced by TGF- $\beta$ in different contexts of fibrotic disease as a specific downstream effector of its activity (Leask et al., 2002). To date, the in vivo silencing of target genes involved also in chronic disease such as fibrosis, including CTGF, can be achieved through various therapeutic strategies, either via local or by means of systemic administration of viral and non-viral vectors. One of the most promising strategy is represented by the gene therapy through the selective gene knock-down mediated by the small interfering RNAs (siRNAs) or the microRNAs (miRNAs) (Lam et al., 2015; Salazar-Montes et al., 2015; Omar et al., 2016). These therapeutic molecules are short non-coding RNAs with a great potential for different clinical applications (Gori et al., 2015; Lam et al., 2015). However, in order to increase the silencing efficiency of siRNAs and miRNAs the search for the most suitable carrier in terms of low toxicity and immunogenicity to target cells remains an open challenge. In such a scenario, NP-based delivery of siRNAs might represent an ideal solution by improving not only the safety of this potential therapy, but also its effectiveness (Surendran et al., 2017; Yu et al., 2020). The main advantages of NPs are their tunable size, shape and surface features along with their adjustable biological properties (Miele et al., 2012). Among the various material formulations tested, including gold, silica, porous silicon, CNT and diverse polymers, magnetic iron oxide NPs seem to be the most interesting for gene therapy due to their reduced toxicity, easy surface modification and high versatility in a wide range of biomedical applications ( Wu et al., 2008;
Xiao et al., 2014; Saeed et al., 2018). In this regard, a research group has recently investigated in vitro the anti-fibrotic activity of polyethyleneimine (PEI)-functionalized magnetic iron oxide NPmediated delivery of siRNAs against CTGF (Yu et al., 2020). The siRNA-loaded NPs showed low cytotoxicity and high transfection efficiency, along with significant CTGF silencing performance, reducing collagen production and deposition in the hepatic stellate cell line LX-2. Thus, taking the cue from this study one could envision the use of invasive electrodes with nanoparticleembedded coatings, such as hydrogels, to regulate the controlled delivery of siRNAs or miRNAs for the specific silencing of CTGF, or other mediators of inflammation and fibrosis.

\section{FURTHER TISSUE ENGINEERING STRATEGIES FOR TARGETED DRUG RELEASE}

Ideally, drug loading within the coating of an implantable neural device with tunable physicochemical characteristics, can help avoid adverse side effects associated to systemic administration thanks to the controlled local delivery of the appropriate amount of the drug and for the desired time-window. In the last decades, a considerable number of brilliant approaches have been attempted in order to dope, absorb and incorporate in the interface coating the desired drug to accomplish a safe, effective, controlled and long-term pharmacological release as the aforementioned examples with dexamethasone. Drug loading into the coating can be realized through a self-assembly procedure, by means of electrostatic interactions, using a charged drug as dopant agent or hydrophobic interactions, via physical entrapment, as well as covalent bonding using degradable peptides or cleavable molecular linkers (Balint et al., 2014; Zeglio et al., 2019). The miniaturization of biomedical devices through the use of microfluidics is a novel opportunity for tuning the properties of flexible and stretchable biomaterials in many smart applications, from biology to medicine and tissue engineering, including drug delivery purposes (Fallahi et al., 2019). Also, novel technologies for long-term encapsulation using new arising materials have recently emerged with promising results. These include: thinfilms of inorganic coatings of $\mathrm{Al}_{2} \mathrm{O}_{3}$ (alumina), $\mathrm{SiO}_{2}$ (silica), $\mathrm{SiC}$ (carborundum) and diamond; in addition, several organic coatings have been used, which are made of - among others - parylene C, polyimide, liquid crystal polymer (LCP), SU-8 and silicone elastomer for implantable microfabricated medical devices. Both chemical solutions, organic and inorganic, leverage on the miniaturization of the implants thanks to the material flexibility and scalability (Ahn et al., 2019). Although the extended suitability - over several decades - of these materials for the encapsulation of biomedical devices has been largely demonstrated in the literature, they have not yet been approved for chronic implantation in patients (Ahn et al., 2019). However, for the prospective chronic encapsulation of microfabricated implants, these novel materials could overcome the performances of conventional macroscale ceramics- or metal-based packaging technologies that offer scarce adaptability to the microfabrication processes; these emerging materials possess indeed largely 
tunable physicochemical properties and higher biocompatibility as well as reliability for clinical applications in order to control the FBR. Nevertheless, much efforts need to be done for their complete processing and engineering, in particular a multicombinational approach with the association of various organic and inorganic materials will be advisable in the next future for developing an optimal deposition process and studying their barrier characteristics (Ahn et al., 2019). In a representative example of such approach, the authors compared the longterm behavior of Utah electrode array-based neural interfaces, encapsulated in a bilayer of $\mathrm{Al}_{2} \mathrm{O}_{3}$ and parylene $\mathrm{C}$, vs. the same electrodes with parylene C-only encapsulation (Xie et al., 2014). They observed higher performance stability (i.e., stable powerup frequencies and constant radio-frequency signal strength) and thus increased lifetime of the bilayer encapsulated devices compared with the parylene-only devices. Moreover, the former represented a more reliable encapsulation method for the functionality of chronically implanted neural interfaces.

As regards the investigation of ideal biomaterials for controlled drug delivery, in an in vitro analysis three different types of carefully designed pHEMA hydrogel coatings were applied to microfabricated neuroprosthetic devices, through specific hydrogel casting methods, with incorporation of lysine and $\mathrm{NaCl}$ to increase both storage capacity and local pharmacological delivery rate in the brain (Jhaveri et al., 2009). Although promising in terms of favorable neural cell response upon Nerve Growth Factor (NGF) delivery, their study needs to be refined for extended in vivo applications. In fact, the mechanical properties of these coatings need to be improved to mimic more closely those of peripheral nervous tissue in order to avoid delamination following their insertion in the body; also, more detailed studies should be planned for determining specific local drug delivery and degradation time in vivo besides the NGF tested herein. In another interesting in vitro investigation on new smart multifunctional biomaterials, electrically-responsive core-shell hybrid microfibers, coated with PEDOT by chemical polymerization, were used for the controlled release of the anti-inflammatory diclofenac sodium salt (Chen et al., 2017). The microfibers were fabricated through a combination of coaxial wet spinning of a hydrogel core of bacterial cellulose, using a microfluidic device, and a dip-coating method of the hydrogel with a conductive polymer shell layer of PEDOT. The developed hybrid microfibers showed very high biocompatibility, electroactivity and allowed the researchers to control the diclofenac release via external electrical stimulation in a rat neural cell line. Instead, a remarkable intervention strategy at relevant time scales for chronic clinical applications was the one proposed by Boehler et al. (2017). They microfabricated flexible layers of polyimide on a Pt-IrOx electrode with subsequent coating of PEDOT to harness its conductive properties and drug delivery capacity, for sustained (12 weeks) dexamethasone delivery in implanted rat brains. The drug was incorporated during the polymerization step of PEDOT and released in a controlled manner for attenuating the FBR over the 12week period that is way beyond the initial healing phase of 6 weeks. Instead, an engineered PEG-maleimide hydrogel coating for neural electrodes was developed to actively control the local release of an anti-inflammatory molecule (IL-1Ra) in vivo (Gutowski et al., 2015). They tuned the physiochemical properties of the hydrogel by developing a stimulus-responsive degradable portion for on-demand release of the anti-inflammatory agent in rat brain tissue. Indeed, by taking advantage of the high expression of matrix metalloproteinases (MMPs) in the inflamed rat brain, the authors functionalized the hydrogel coating with MMP-degradable crosslinking peptides that were able to release IL-1Ra at the brain-implant interface in response to inflammation. Altogether, they observed only a moderate reduction of inflammatory markers, although neuronal survival around the electrodes was higher than uncoated controls. However, further improvements are necessary to verify the efficacy of this strategy also in peripheral nerves and for chronic implants, such as a reduced adhesion to the coating of other cell types besides glial and neuronal cells. Importantly, since no evident differences were detected in the recruitment and activation of inflammatory cells involved in scar formation between coated and uncoated implants, it will be of utmost importance to work more on this aspect. Another interesting avenue leverages the properties of conducting and conjugated polymer-based devices to create a drug-eluting electrode. The device can be loaded with the drug of choice and the release is electronically triggered by electrostatic interactions and/or electrical stimulation. In one of these studies, the drug of interest was entrapped in an electropolymerized PEDOT:PSS film by means of a gentle supercritical carbon dioxide $\left(\mathrm{scCO}_{2}\right)$ treatment and then gradually released in vitro via electrical stimulation, retaining an elevated activity while maintaining normal electrochemical properties of the polymer surface (Löffler et al., 2016). Such $\mathrm{scCO}_{2}$-based method could represent a smart approach for loading anionic and cationic drugs in any conductive bio-coating, which can be adjusted depending on the purpose. A different strategy that exploited the mixed conductivity of PEDOT:PSS is the one based on implantable ion pumps (Isaksson et al., 2007). Such technological platform can be potentially utilized not only for targeted ion delivery, but also for larger biomolecules, such as glutamate, aspartate and y-aminobutyric acid (GABA) (Simon et al., 2009), which can be particularly useful for the treatment of CNS disorders. For example, a clinically relevant in vivo application of an ion pump for controlled GABA delivery was carried out in rat models of peripheral nerve injury (Jonsson et al., 2015). In this study, a specific design of the outlets of the implantable organic electronic delivery device was developed for local GABA release along the spinal cord. It showed the ability to mitigate neuropathic pain with no side effects.

A recent advancement of this technology for in vivo applications, was the development of a microfluidic ion pump with high drug-delivery ability (Uguz et al., 2017). The major advantage of this novel configuration was represented by the reduced distance for the electrophoretic transport of the drug, requiring a low voltage for its delivery, and by the fact that the microfluidic channels were connected to an almost inexhaustible drug reservoir. A similar microfluidic ion device with PEDOT:PSS-based recording electrodes was fabricated for releasing GABA in a specific brain region of an epilepsy 
mouse model (Proctor et al., 2018). The device, composed by a neural probe incorporating a microfluidic ion pump and neural electrodes for recording neural activity, allowed the inhibitory neurotransmitter to be selectively delivered to the seizure source for its control and termination. Accordingly, these ion pump devices may represent very interesting spatially and temporally controlled electrophoretic drug delivery systems. These implanted platforms may hold tremendous potential for on-demand therapeutic drug delivery also when combined to intraneural electrodes. The authors speculate that their device could be useful also in chronic drug delivery settings for reducing the FBR after additional technological developments, especially related to the drug reservoir reloading and the improvement of their long-term biostability.

Other investigators generated graphene oxide (GO) nanocomposite films, deposited inside a conducting PPy scaffold, to enhance the dexamethasone phosphate-loading capacity of the graphene component, by means of physical adsorption, and to elicit its electrically controlled delivery (Weaver et al., 2014). In vitro tests carried out in primary rat astrocytes showed the possibility to finely control and adjust the drug-release time and dosage, depending on the need, by varying the ultrasonication time required to prepare the graphene oxide nanosheets. Thereby, modulating the ultrasonication treatment one can influence the film morphology, drug load and release profile in a versatile manner. However, many of these smart tissue engineering strategies have failed to reach patients' bed because of a series of drawbacks. Among them, it is worth considering the frequent lack of a suitably charged dopant molecule and the poor drug loading performance with low and unsatisfactory concentration, particularly when using conjugated and conductive polymeric films. Besides, additional hurdles to be addressed include film instability and drug leakage. In particular, the undesired leakage of the drug from the polymeric coating may indeed occur when the loaded molecule is too small compared to the pore size of the releasing hydrogel (Zeglio et al., 2019). As for the limitation of poor drug loading performance, mostly for delivering large molecule therapeutics, it can be addressed through the use of NPs made of conductive polymers (such as PPy) (Hosseini-Nassab et al., 2017). Thanks to their higher surface area than conventional conductive thin films, the electroresponsive PPy NPs enabled a controlled and efficient release of surface-loaded bioactive insulin, triggered by electrical stimuli on a coated Pt electrode, also in in vivo tests of therapeutic delivery in mouse models. The authors speculate that such drug-loaded NPs may be enclosed into a semi-permeable hydrogel coating that allows only drug molecules to pass through. In conclusion, this strategy could be potentially envisioned also for peripheral neural interfaces. Such implantable drug delivery system could improve spatially and temporally controlled drug release by simply varying the ratio of the quantity of NPs to the concentration of the desired drug, while maintaining its bioactivity.

Lastly, alternative and smart methods for interfering with the interaction between device surface and tissue microenvironment, thereby evading the host immune response, could be represented by: (a) modifications of biomaterial surface with adhesive peptides (e.g., RGD cell adhesion ligand on PEG surfaces) to partially attenuate inflammatory reaction and capsule formation (Lynn et al., 2011); (b) functionalization of PEG-coated surfaces with synthetic human-based "self" peptides (e.g., the immunomodulatory membrane proteins CD47 and CD200) to inhibit macrophage-mediated clearance of the surface and prolong its in vivo survival (Rodriguez et al., 2013; Kim et al., 2014).

A summary of the intervention strategies based on the control of the interface-microenvironment interaction with representative examples is reported in Table 3.

\section{FINAL REMARKS AND FUTURE DIRECTIONS}

To date, the design of resolutive solutions to modulate the FBR, based on the exhaustive comprehension of its molecular mechanisms, represents a major challenge for a suitable and long-lasting implantation of intraneural devices.

Modern neuroprostheses may employ electrodes produced with microtechnology that, however, do not go below the size of some tens of micrometers. Various techniques of micromachining and micromolding of flexible and conductive polymer coatings may allow scientists to fine tuning the features of the electrodes to the characteristics of the host tissue, thus creating more stable devices over time. Furthermore, the integration of microfluidic ion pumps and channels into neural probes can be harnessed for extended drug delivery in the implanted tissue, so to dramatically reduce the FBR and to be much better tolerated than plain implants.

Indeed, microfluidics and, most of all, nanofluidics, although promising are still quite unexplored in neuroprosthetics, and deserve further investigation.

Strategies based on microfluidic, microscale and nanoscale technologies provide scalability. They can lead from the longterm and stable neurotransmission simultaneously to many tissue points, to an enhancement of spatial selectivity stimulation, through implantable microelectrode arrays and microscale actuators (Kozai, 2018; Kumar et al., 2020). Even more so because conventional electrodes and recording systems are bulky and unsuitable for single cell resolution. By micro- and nano-engineering the surface properties of the implant, one can obtain a better control of therapeutic drug release from artificial nanopores, NPs made of conductive polymers and other nanostructured materials. Compared to traditional bulk materials, this latter mechanism can take advantage of the higher surface area of loaded NPs, their variable degradation rate depending on the biomaterial used, and the adjustable selectivity and permeability of hydrogel coatings to drug molecules. Biofunctionalization of NPs with antigen-recognized antibodies may further ameliorate targeting efficiency by increasing the drug concentration within a specific tissue (Cai and $\mathrm{Xu}, 2011$ ).

For example, bioinspired cellulose nanocomposites have higher versatility and functionality than rigid silicone implants, due to their switchable stiffness characteristics, reducing neuroinflammation in chronic implants (Nguyen et al., 2014). 
TABLE 3 | Intervention strategies based on the control of the interface-microenvironment interaction.

(iii) Interface-microenvironment interaction

\begin{tabular}{ll}
\hline Intervention strategy & Examples \\
\hline $\begin{array}{l}\text { Targeted drug delivery systems } \\
\text { Anti-inflammatory drugs }\end{array}$ & $\begin{array}{l}\text { Extended overview } \\
\text { Dexamethasone }\end{array}$ \\
& \\
& IL-1Ra \\
& Ibuprofen \\
& Clodronate liposomes \\
& Diclofenac \\
& \\
& RGD cell adhesion ligands on glass and \\
& PEG surfaces \\
& Functionalization of PEG surfaces with \\
& human self-peptides \\
& Extended overview \\
& Targeted silencing of CTGF via siRNAs-, \\
& miRNAs- and nanoparticle-based silencing \\
\end{tabular}

\section{References}

Reviewed in Cai and Xu (2011)

Reviewed in Lotti et al. (2017) and Zeglio et al. (2019)

Spataro et al., 2005; Kim and Martin, 2006; Wadhwa et al., 2006; Mercanzini et al., 2010; Luo et al., 2011; Weaver et al., 2014; Alba et al., 2015; Boehler et al., 2017; de la Oliva et al., 2018b, 2019; Kleber et al., 2019

Gutowski et al., 2015)

de la Oliva et al., 2018b

de la Oliva et al., 2018b

Reviewed in Zeglio et al. (2019)

Chen et al., 2017

Reviewed in Zeglio et al. (2019)

Anderson et al., 1999; Lynn et al., 2011; Blakney et al., 2012

Reviewed in Lotti et al. (2017)

Kim et al., 2014

Reviewed in Lotti et al. (2017)

Reviewed in Leask et al. (2002); Wu et al. (2008), Miele et al. (2012); Gori et al. (2015), Salazar-Montes et al. (2015); Omar et al. (2016), Surendran et al. (2017), and Saeed et al. (2018)

Xiao et al., 2014; Yu et al., 2020

CSF1R inhibition

Doloff et al., 2017

Tissue engineering strategies Extended overview for targeted drug release

Human self-peptides
Conductive polymer films
Electrically-responsive microfibers
Milliscale dynamic soft reservoir (DSR)
Embedded microfluidic channels
Hydrogel coating (e.g., pHEMA,
PEG-maleimide, PVA-heparin)
CNTs nanoreserviors
Electronic ion pumps
Microencapsulation
Nanoparticle-based delivery

Electrically controlled drug delivery from graphene oxide nanocomposite film of PPy
Reviewed in Ratner (2002); Drury and Mooney (2003), Knop et al. (2010); Balint et al. (2014), Gori et al. (2015); Lam et al. (2015), Salazar-Montes et al. (2015), and Zeglio et al. (2019)

Rodriguez et al., 2013

Wadhwa et al., 2006; Mario Cheong et al., 2014; Löffler et al., 2016

Chen et al., 2017

Dolan, 2019

Metz et al., 2004; Retterer et al., 2004; Ziegler et al., 2006; Gao et al., 2013; Takehara et al., 2014; Minev et al., 2015; Elyahoodayan et al., 2020

Jhaveri et al., 2009; Mario Cheong et al., 2014; Gutowski et al., 2015

Luo et al., 2011

Isaksson et al., 2007; Simon et al., 2009; Jonsson et al., 2015; Uguz et al., 2017; Proctor et al., 2018

Campioni et al., 1998

Reviewed in Wu et al. (2008); Cai and Xu (2011), Miele et al. (2012); Surendran et al. (2017), and Saeed et al. (2018)

Kim and Martin, 2006; Xiao et al., 2014; Hosseini-Nassab et al., 2017; Yu et al., 2020

Weaver et al., 2014

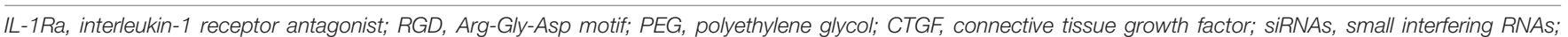

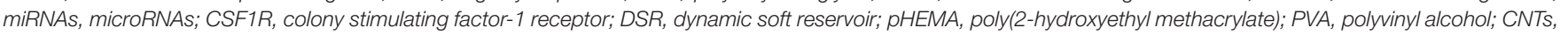
carbon nanotubes; PPy, polypyrrole. References: except were specifically indicated as 'Reviewed in,' all others are research articles.

Indeed, these mechanically adaptive nanomaterials although initially rigid become compliant after intracortical implantation in rats. They have been proven to lower neuroinflammatory response at chronic time-points, with no neuronal loss, limited scarring, reduced blood-brain barrier damage as well as decreased accumulation of activated microglia and macrophages at the implant-tissue interface. Upon insertion in the brain, when exposed to physiological conditions, the nanocomposites exhibited a massive reduction in tensile storage modulus and, in turn, the induced tissue strain was dramatically lowered (Nguyen et al., 2014).
Moreover, nanofiber-formed nanogels and self-assembly nanoscaffold hydrogels are broadly adopted for targeted and controlled drug delivery. For instance, some antibody-drug conjugate payloads can be maintained in a target area by side chains, chemical moieties and interactions with the nanogel, thus prolonging their protective effect (Cai and $\mathrm{Xu}, 2011$ ).

Taking into consideration all of the above reviewed biomedical strategies, ultraflexible nanosized devices, coated with biocompatible and mechanically dynamic materials, may represent an optimal solution. Owing to their advantageous features of stiffness/compliance in a neural context, such 
devices seem to be able to significantly attenuate the intraneural invasiveness, tissue strain, micromotion stress and, in turn, the chronic inflammatory response of the tissue.

It is worth noting, though, that there still remain several technical snags that must be overcome in the manufacturing of suitable nanofluidic components over the next decade. Because of its novelty, expertise in nanofluidics is not as robust as the one in microfluidics and it lacks standardized procedures for the fabrication of neural nanodevices. So, manufacturing accuracy of neural nanointerfaces still depends a lot on the ability of the single producer.

However, current fabrication technologies of advanced neural electrodes that combine the employment of new CPs with complex micro- and nano-structured configurations, such as fluidic microdrives, are based on rapidly growing micro- and nano-electronics expertise. These novel methodologies permit the development and use of flexible and small-sized devices with more targeted stimulation by applying low voltages in a safe manner. Therefore, they allow researchers to obtain very good neural signals while reducing the implant invasiveness and its mechanical deformation (Gerwig et al., 2012; Lorach et al., 2015; Samba et al., 2015; Bazard et al., 2017; Qi et al., 2017; Vitale et al., 2018). Furthermore, to match the mechanical texture of the neural tissue, especially of the brain, conductive and ultraflexible nanomaterials, such as CNTs, ultrathin films of graphene and nanowires have been explored. Such highly flexible and compliant electrodes can thus bend and adapt to the movements of the host tissue only in a slightly invasive and detrimental manner. Nevertheless, various methods for the implantation into the neural tissue of these nanofabricated devices require temporary stiffening factors that sometimes tend to augment the electrode size and stiffness, thereby increasing also tissue damage, cell death and eventually giving rise to a severe and unwanted inflammatory response (Vitale et al., 2018).

Hitherto, the majority of these studies that investigate alternative strategies against the FBR have been carried out either on the CNS or using other cell types in vitro, such as cardiac cells. Hence, many efforts have yet to be done to achieve suitable solutions also in peripheral nerves. Despite these hurdles, we believe that the challenge of ensuring a high-resolution release of bioactive chemicals against the FBR by minimally invasive neural interfaces while, at the same time, precisely controlling neurostimulation will need nanofluidics to be fully accomplished. Indeed, the smaller the size of the invasive electrode with an associated lower stiffness, the better the response of the neural tissue and the more selective and tailored will be the control over the device functionality. Among the main advantages for the use of nanoelectrodes, there is undoubtedly the enhanced mass transport, favored by the reduced dimensions, which determines an increased flow of Faradaic currents (Kotov et al., 2009). Another plus is represented by the increased spatial resolution of neural stimulation compared to microelectrodes (Fattahi et al., 2014), and the possibility to miniaturize several parallel nanoelectrodes within the same device, so to be used for simultaneous multiplexed measurements (Wang M. et al., 2018). Indeed, a more accurate control of the structural features of neural interfaces at subcellular level, with improved electrical properties, is preferable for neural recordings in vivo, while limiting detrimental side effects.

However, the chronic use of such invasive, although soft and small-sized electrodes, for neuroprosthetic applications will require biochemical functionalization of the surface with biocompatible coatings. These could be bioactive moieties (e.g., specific chemical signals from peptide epitopes) incorporated within conductive polymers, leveraging their tunable physicochemical properties that provide a wide versatility of intervention. A detailed investigation on how the nanotopography modifications and the chemical reactive potential of the surface can reduce plasma protein adsorption and immune cell adhesion will help control the inflammatory response. In addition, the controlled and continuous release of neurotrophic factors and the targeted delivery of therapeutic drugs to further improve the biological response to the implant and avoid the FBR will be of paramount importance. Accordingly, tailoring zwitterionic hydrogels to incorporate bioactive materials, such as ECM-derived organic components (e.g., RGD motifs that mediate the cell-fibronectin attachment) or neural cells will be pivotal (Von Der Mark et al., 2010). In this regard, it could be envisioned as particularly appealing a cell-based co-therapy, with the integration of autologous neural cells or patient-specific induced pluripotent stem cell (iPSC)-derived neural cells into the interface coating so to escape their recognition by the host immune system, hampering the consequent inflammatory cascade (Xu et al., 2013; Amin et al., 2016). Together with these solutions, loading the hydrogel with selected chemicals and drugs either linked to the surface or encapsulated into novel NPs seem the best routes to take (Aregueta-Robles et al., 2014; Lotti et al., 2017). Also, diffusionmediated delivery systems based on micro-optical fluidic devices and microfluidic channels integrated into neural interfaces may represent another valid intervention strategy for a controlled release of therapeutics in chronic implants (Retterer et al., 2004; Takehara et al., 2014). These newly developed microfluidic devices have been characterized only in vitro and in vivo, though in mouse brain and in chronically implanted intracortical probes in rats, but they have been proven to be effective in controlling and hindering reactive responses in neural cells and brain tissue.

How limiting the leakage and the exhaustion of the drug payload in the microenvironment around the electrode site? This question still remains an open issue that the implementation of surface micromachining for the synthesis of NPs as ideal drug carriers, and microfluidic technologies could likely solve in the next years. For instance, integration and modification of the electrode surface through MEMS devices, microactuators and DSRs with permeable actuating membranes, may at least help control the pharmacological release and limit the drug leakage.

It is well known that biomaterials or tissue-engineered constructs can strongly influence the interactions between a foreign body and the host immune system. Therefore, the deposition technology of the most appropriate coating on the invasive electrode and its modification with biomimetic surfaces targeted to support tissue-specific cell functions will pave the way to the definitive solution for mitigating the FBR and for advancing the long-term use of neural-interfaced prostheses. 
It follows that, before in vivo testing, the choice of the best biomimetic coating to be used will rely on preliminary results gained from complex in vitro co-culture systems, as Lab-ona-Chip devices. The latter should indeed be developed so as to recapitulate more closely all biological aspects of the intricate tissue damage, vascular injury and inflammatory cascade associated with electrode implantation. Thus, leveraging on complex microfluidic and, hopefully, nanofluidic co-culture platforms for mimicking both nervous tissue microenvironment, with patient-specific cell types, and the implant-induced FBR, one could preliminarily analyze the tissue response to a certain biomaterial coating in a physiologically relevant manner (Sharifi et al., 2019). Such opportunity raises the need for a strict collaboration between medical sciences and bioengineering. The former are necessary for having a detailed knowledge on specific mechanisms and timing of adsorption of the host proteins and cells on the implant surface; bioengineering expertise and technologies are instead essential to reproduce and simulate the entire environment, behavior and physiological responses of the nervous tissue to biomaterials. Additional work will be required to identify exactly and control the biological mechanisms of the wound healing process, and shed light on the causal connections between mechanical, chemical, immunological and inflammatory events underlying the acute and chronic peripheral nerve response. Notably, the important role played by the bloodnerve barrier must be better investigated, whose stability can be compromised by the traumatic event of the device insertion (Stubbs, 2020).

A further technical enrichment for increasing a priori our knowledge on the mechanisms underlying the development of the FBR for a more effective electrode engineering, comes from the use of in silico methods. In this respect, a very illuminating example showed a data-driven approach based on polynomial functions to simulate and investigate the development of scar tissue outgrowth around an implanted neural device over time (Sergi et al., 2020). Such computer-based approach could be combined with micro/nanofabrication and biochemical functionalization techniques for having a more representative prediction of the possible fibrous capsule consistency before collecting

\section{REFERENCES}

Abidian, M. R., Corey, J. M., Kipke, D. R., and Martin, D. C. (2010). Conductingpolymer nanotubes improve electrical properties, mechanical adhesion, neural attachment and neurite outgrowth of neural electrodes. Small 6, 421-429. doi: 10.1002/smll.200901868

Ahn, S. H., Jeong, J., and Kim, S. J. (2019). Emerging encapsulation technologies for long-term reliability of microfabricated implantable devices. Micromachines 10:508. doi: 10.3390/mi10080508

Alba, N. A., Du, Z. J., Catt, K. A., Kozai, T. D. Y., and Cui, X. T. (2015). In vivo electrochemical analysis of a PEDOT/MWCNT neural electrode coating. Biosensors 5, 618-646. doi: 10.3390/bios5040618

Altun, A., Apetrei, R. M., and Camurlu, P. (2019). The effect of copolymerization and carbon nanoelements on the performance of poly(2,5-di(thienyl)pyrrole) biosensors. Mater. Sci. Eng. C 105:110069. doi: 10.1016/j.msec.2019.110069

Ambrico, M., Ambrico, P. F., Cardone, A., Cicco, S. R., Palumbo, F., Ligonzo, T., et al. (2014). Melanin-like polymer layered on a nanotextured silicon surface experimental data, thereby helping scientists in the choice of the most suitable surface coating against the development of the FBR (Di Pino et al., 2010). Overall, addressing these interesting challenges will require a close interaction between neuroengineering and biology on multiple levels for producing and, once inserted, stabilizing cutting-edge neural interfaces, and thus responding to the requests of the clinical therapy.

\section{AUTHOR CONTRIBUTIONS}

MG conceived the idea, wrote the manuscript, and prepared figures and tables. GV supervised and revised the manuscript. SG contributed to wrote the manuscript and prepared the tables. VD supervised the manuscript preparation. GD contributed to wrote, supervised, revised, and critically discussed the manuscript. All authors contributed to the article and approved the submitted version.

\section{FUNDING}

Funding for the open access publication fees was provided in the framework of the joint projects: WiFi Myo-Hand "Sistema impiantabile ottimizzato per l'interfacciamento con il sistema nervoso periferico e il controllo della protesi di arto superiore" and "RGM5: (re)-Give me five, exploring new frontiers in prosthetic surgery" between Università Campus Bio-Medico di Roma (UCBM) and Istituto Nazionale per l'Assicurazione contro gli Infortuni sul Lavoro (INAIL).

\section{ACKNOWLEDGMENTS}

The authors acknowledge the financial support from Istituto Nazionale per l'Assicurazione contro gli Infortuni sul Lavoro (INAIL). MG dedicates this review article to Agnese for her loving commitment to our kids and the resilience shown during the harsh lockdown due to the COVID-19 pandemic.

for a hybrid biomimetic interface. J. Mater. Chem. C 2, 573-582. doi: 10.1039/ c3tc31327a

Ambrico, M., Ambrico, P. F., Cardone, A., Della Vecchia, N. F., Ligonzo, T., Cicco, S. R., et al. (2013). Engineering polydopamine films with tailored behaviour for next-generation eumelanin-related hybrid devices. J. Mater. Chem. C 1, 1018-1028. doi: 10.1039/c2tc00480a

Amin, H., Maccione, A., Marinaro, F., Zordan, S., Nieus, T., and Berdondini, L. (2016). Electrical responses and spontaneous activity of human iPS-derived neuronal networks characterized for 3-month culture with 4096-electrode arrays. Front. Neurosci. 10:121. doi: 10.3389/fnins.2016.00121

Anderson, H. E., and Weir, R. F. (2019). On the development of optical peripheral nerve interfaces. Neural Regen. Res. 14, 425-436. doi: 10.4103/1673-5374. 245461

Anderson, J. M. (2000). Multinucleated giant cells. Curr. Opin. Hematol. 7, 40-47. doi: 10.1097/00062752-200001000-00008

Anderson, J. M., Defife, K., Mcnally, A., Collier, T., and Jenney, C. (1999). Monocyte, macrophage and foreign body giant cell interactions with 
molecularly engineered surfaces. J. Mater. Sci. Mater. Med. 10, 579-588. doi: 10.1023/a:1008976531592

Anderson, J. M., Rodriguez, A., and Chang, D. T. (2008). Foreign body reaction to biomaterials. Semin. Immunol. 20, 86-100. doi: 10.1016/j.smim.2007.11.004

Andrade, J. D., and Hlady, V. (1987). Plasma protein adsorption: the big twelve. Ann. N. Y. Acad. Sci. 516, 158-172. doi: 10.1111/j.1749-6632.1987.tb33 038. $\mathrm{x}$

Aregueta-Robles, U. A., Woolley, A. J., Poole-Warren, L. A., Lovell, N. H., and Green, R. A. (2014). Organic electrode coatings for next-generation neural interfaces. Front. Neuroeng. 7:15. doi: 10.3389/fneng.2014.00015

Armstrong, D. A., Major, J. A., Chudyk, A., and Hamilton, T. A. (2004). Neutrophil chemoattractant genes KC and MIP- 2 are expressed in different cell populations at sites of surgical injury. J. Leukoc. Biol. 75, 641-648. doi: 10.1189/jlb.0803370

Arreaga-Salas, D. E., Avendaño-Bolívar, A., Simon, D., Reit, R., Garcia-Sandoval, A., Rennaker, R. L., et al. (2015). Integration of high-charge-injection-capacity electrodes onto polymer softening neural interfaces. ACS Appl. Mater. Interf. 7 , 26614-26623. doi: 10.1021/acsami.5b08139

Ashcroft, G. S. (1999). Bidirectional regulation of macrophage function by TGF- $\beta$. Microb. Infect. 1, 1275-1282. doi: 10.1016/S1286-4579(99)00257-9

Badia, J., Boretius, T., Andreu, D., Azevedo-Coste, C., Stieglitz, T., and Navarro, X. (2011). Comparative analysis of transverse intrafascicular multichannel, longitudinal intrafascicular and multipolar cuff electrodes for the selective stimulation of nerve fascicles. J. Neural Eng. 8:36023. doi: 10.1088/1741-2560/ $8 / 3 / 036023$

Badia, J., Raspopovic, S., Carpaneto, J., Micera, S., and Navarro, X. (2016). Spatial and functional selectivity of peripheral nerve signal recording with the transversal intrafascicular multichannel electrode (TIME). IEEE Trans. Neural Syst. Rehabil. Eng. 24, 20-27. doi: 10.1109/TNSRE.2015.2440768

Balint, R., Cassidy, N. J., and Cartmell, S. H. (2014). Conductive polymers: towards a smart biomaterial for tissue engineering. Acta Biomater. 10, 2341-2353. doi: 10.1016/j.actbio.2014.02.015

Banks, R. E., Forbes, M. A., Kinsey, S. E., Stanley, A., Ingham, E., Walters, C., et al. (1998). Release of the angiogenic cytokine vascular endothelial growth factor (VEGF) from platelets: significance for VEGF measurements and cancer biology. Br. J. Cancer 77, 956-964. doi: 10.1038/bjc.1998.158

Bazard, P., Frisina, R. D., Walton, J. P., and Bhethanabotla, V. R. (2017). Nanoparticle-based plasmonic transduction for modulation of electrically excitable cells. Sci. Rep. 7:7803. doi: 10.1038/s41598-017-08141-4

Bellingan, G. J. (1996). In vivo fate of the inflammatory macrophage during the resolution of inflammation: inflammatory macrophages do not die locally, but emigrate to the draining lymph nodes. J. Immunol. 157, 2577-2585.

Blakney, A. K., Swartzlander, M. D., and Bryant, S. J. (2012). Student award winner in the undergraduate category for the society of biomaterials 9th World Biomaterials Congress, Chengdu, China, June 1-5, 2012: the effects of substrate stiffness on the in vitro activation of macrophages and in vivo host response to poly(ethylene glycol)-based hydrogels. J. Biomed. Mater. Res. Part A 100 A, 1375-1386. doi: 10.1002/jbm.a.34104

Blau, A., Murr, A., Wolff, S., Sernagor, E., Medini, P., Iurilli, G., et al. (2011). Flexible, all-polymer microelectrode arrays for the capture of cardiac and neuronal signals. Biomaterials 32, 1778-1786. doi: 10.1016/j.biomaterials.2010. 11.014

Bochenek, M. A., Veiseh, O., Vegas, A. J., McGarrigle, J. J., Qi, M., Marchese, E., et al. (2018). Alginate encapsulation as long-term immune protection of allogeneic pancreatic islet cells transplanted into the omental bursa of macaques. Nat. Biomed. Eng. 2, 810-821. doi: 10.1038/s41551-018-0275-1

Boddupalli, A., Zhu, L., and Bratlie, K. M. (2016). Methods for implant acceptance and wound healing: material selection and implant location modulate macrophage and fibroblast phenotypes. Adv. Healthc. Mater. 5, 25752594. doi: $10.1002 /$ adhm. 201600532

Boehler, C., Kleber, C., Martini, N., Xie, Y., Dryg, I., Stieglitz, T., et al. (2017). Actively controlled release of Dexamethasone from neural microelectrodes in a chronic in vivo study. Biomaterials 129, 176-187. doi: 10.1016/j.biomaterials. 2017.03.019

Bottini, M., Bruckner, S., Nika, K., Bottini, N., Bellucci, S., Magrini, A., et al. (2006). Multi-walled carbon nanotubes induce T lymphocyte apoptosis. Toxicol. Lett. 160, 121-126. doi: 10.1016/j.toxlet.2005.06.020

Branner, A., Stein, R. B., Fernandez, E., Aoyagi, Y., and Normann, R. A. (2004). Long-term stimulation and recording with a penetrating microelectrode array in cat sciatic nerve. IEEE Trans. Biomed. Eng. 51, 146-157. doi: 10.1109/TBME. 2003.820321

Brummer, S. B., Robblee, L. S., and Hambrecht, F. T. (1983). Criteria for selecting electrodes for electrical stimulation: theoretical and practical considerations. Ann. N. Y. Acad. Sci. 405, 159-171. doi: 10.1111/j.1749-6632.1983.tb31628.x

Cai, X. J., and $\mathrm{Xu}$, Y. Y. (2011). Nanomaterials in controlled drug release. Cytotechnology 63, 319-323. doi: 10.1007/s10616-011-9366-5

Campioni, E. G., Nobrega, J. N., and Sefton, M. V. (1998). HEMA/MMMA microcapsule implants in hemiparkinsonian rat brain: biocompatibility assessment using [3H]PK11195 as a marker for gliosis. Biomaterials 19, 829837. doi: 10.1016/S0142-9612(97)00241-X

Canales, A., Jia, X., Froriep, U. P., Koppes, R. A., Tringides, C. M., Selvidge, J., et al. (2015). Multifunctional fibers for simultaneous optical, electrical and chemical interrogation of neural circuits in vivo. Nat. Biotechnol. 33, 277-284. doi: 10.1038/nbt.3093

Carli, S., Bianchi, M., Zucchini, E., Di Lauro, M., Prato, M., Murgia, M., et al. (2019). Electrodeposited PEDOT:Nafion composite for neural recording and stimulation. Adv. Healthc. Mater. 8:e1900765. doi 10.1002/adhm.2019 00765

Carr, L. R., Zhou, Y., Krause, J. E., Xue, H., and Jiang, S. (2011). Uniform zwitterionic polymer hydrogels with a nonfouling and functionalizable crosslinker using photopolymerization. Biomaterials 32, 6893-6899. doi: 10. 1016/j.biomaterials.2011.06.006

Castagnola, E., Maggiolini, E., Ceseracciu, L., Ciarpella, F., Zucchini, E., De Faveri, S., et al. (2016). pHEMA encapsulated PEDOT-PSS-CNT microsphere microelectrodes for recording single unit activity in the brain. Front. Neurosci. 10:151. doi: 10.3389/fnins.2016.00151

Chang, K. C., Lu, H. I., Peng, C. W., Lai, M. C., Hsu, S. C., Hsu, M. H., et al. (2013). Nanocasting technique to prepare lotus-leaf-like superhydrophobic electroactive polyimide as advanced anticorrosive coatings. ACS Appl. Mater. Interf. 5, 1460-1467. doi: 10.1021/am3029377

Chang, T. M. S. (1964). Semipermeable microcapsules. Science 146, 524-525. doi: $10.1126 /$ science.146.3643.524

Charkhkar, H., Knaack, G. L., Mchail, D. G., Mandal, H. S., Peixoto, N., Rubinson, J. F., et al. (2016). Chronic intracortical neural recordings using microelectrode arrays coated with PEDOT-TFB. Acta Biomater. 32, 57-67. doi: 10.1016/j. actbio.2015.12.022

Chatzimichail, S., Supramaniam, P., Ces, O., and Salehi-Reyhani, A. (2018). Micropatterning of planar metal electrodes by vacuum filling microfluidic channel geometries. Sci. Rep. 8:14380. doi: 10.1038/s41598-018-32706-6

Chen, C., Chen, X., Zhang, H., Zhang, Q., Wang, L., Li, C., et al. (2017). Electricallyresponsive core-shell hybrid microfibers for controlled drug release and cell culture. Acta Biomater. 55, 434-442. doi: 10.1016/j.actbio.2017.04.005

Chen, S., Jones, J. A., Xu, Y., Low, H. Y., Anderson, J. M., and Leong, K. W. (2010). Characterization of topographical effects on macrophage behavior in a foreign body response model. Biomaterials 31, 3479-3491. doi: 10.1016/j.biomaterials. 2010.01.074

Chen, S., Zheng, J., Li, L., and Jiang, S. (2005). Strong resistance of phosphorylcholine self-assembled monolayers to protein adsorption: insights into nonfouling properties of zwitterionic materials. J. Am. Chem. Soc. 127, 14473-14478. doi: 10.1021/ja054169u

Crowe, M. J., Doetschman, T., and Greenhalgh, D. G. (2000). Delayed wound healing in immunodeficient TGF- $\beta 1$ knockout mice. J. Invest. Dermatol. 115, 3-11. doi: 10.1046/j.1523-1747.2000.00010.x

Cui, X., Wiler, J., Dzaman, M., Altschuler, R. A., and Martin, D. C. (2003). In vivo studies of polypyrrole/peptide coated neural probes. Biomaterials 24, 777-787. doi: 10.1016/S0142-9612(02)00415-5

de la Oliva, N., Del Valle, J., Delgado-Martinez, I., Mueller, M., Stieglitz, T., and Navarro, X. (2019). Long-term functionality of transversal intraneural electrodes is improved by dexamethasone treatment. IEEE Trans. Neural Syst. Rehabil. Eng. 27, 457-464. doi: 10.1109/TNSRE.2019.2897256

de la Oliva, N., Mueller, M., Stieglitz, T., Navarro, X., and Del Valle, J. (2018a). On the use of Parylene C polymer as substrate for peripheral nerve electrodes. Sci. Rep. 8:5965. doi: 10.1038/s41598-018-24502-z

de la Oliva, N., Navarro, X., and del Valle, J. (2018b). Dexamethasone reduces the foreign body reaction to intraneural electrode implants in the peripheral nerve of the rat. Anat. Rec. 301, 1722-1733. doi: 10.1002/ar.2 3920 
de la Oliva, N., Navarro, X., and del Valle, J. (2018c). Time course study of long-term biocompatibility and foreign body reaction to intraneural polyimidebased implants. J. Biomed. Mater. Res. Part A 106, 746-757. doi: 10.1002/jbm.a. 36274

Delgado-Martínez, I., Righi, M., Santos, D., Cutrone, A., Bossi, S., D’Amico, S., et al. (2017). Fascicular nerve stimulation and recording using a novel doubleaisle regenerative electrode. J. Neural Eng. 14:aa6bac. doi: 10.1088/1741-2552/ aa6bac

Deuschl, G., Schade-Brittinger, C., Krack, P., Volkmann, J., Schäfer, H., Bötzel, K., et al. (2006). A randomized trial of deep-brain stimulation for Parkinson's disease. N. Engl. J. Med. 355, 896-908. doi: 10.1056/nejmoa060281

Di Pino, G., Denaro, L., Vadalà, G., Marinozzi, A., Tombini, M., Ferreri, F., et al. (2014). Invasive neural interfaces: the perspective of the surgeon. J. Surg. Res. 188, 77-87. doi: 10.1016/j.jss.2013.12.014

Di Pino, G., Formica, D., Lonini, L., Accoto, D., Benvenuto, A., Micera, S., et al. (2010). ODEs model of foreign body reaction around peripheral nerve implanted electrode. Annu. Int. Conf. IEEE Eng. Med. Biol. Soc. 2010, 15431546. doi: 10.1109/IEMBS.2010.5626825

Di Pino, G., Porcaro, C., Tombini, M., Assenza, G., Pellegrino, G., Tecchio, F., et al. (2012). A neurally-interfaced hand prosthesis tuned inter-hemispheric communication. Restor. Neurol. Neurosci. 30, 407-418. doi: 10.3233/RNN2012-120224

Diao, W., Wu, L., Ma, X., Zhuang, Z., Li, S., Bu, X., et al. (2019). Highly stretchable, ionic conductive and self-recoverable zwitterionic polyelectrolytebased hydrogels by introducing multiple supramolecular sacrificial bonds in double network. J. Appl. Polym. Sci. 136:47783. doi 10.1002/app. 47783

DiPietro, L. A., Burdick, M., Low, Q. E., Kunkel, S. L., and Strieter, R. M. (1998). Mip- $1 \alpha$ as a critical macrophage chemoattractant in murine wound repair. J. Clin. Invest. 101, 1693-1698. doi: 10.1172/JCI1020

Dolan, A. (2019). Title an actuatable soft reservoir modulates host foreign body response Publication Information. Sci. Robot. 4:eaax7043. doi: 10.1126/ scirobotics.aax7043

Doloff, J. C., Veiseh, O., Vegas, A. J., Tam, H. H., Farah, S., Ma, M., et al. (2017). Colony stimulating factor-1 receptor is a central component of the foreign body response to biomaterial implants in rodents and non-human primates. Nat. Mater. 16, 671-680. doi: 10.1038/nmat4866

Drury, J. L., and Mooney, D. J. (2003). Hydrogels for tissue engineering: scaffold design variables and applications. Biomaterials 24, 4337-4351. doi: 10.1016/ S0142-9612(03)00340-5

Du, Z. J., Bi, G. Q., and Cui, X. T. (2018). Electrically controlled neurochemical release from dual-layer conducting polymer films for precise modulation of neural network activity in rat barrel cortex. Adv. Funct. Mater. 28:1703988. doi: 10.1002/adfm.201703988

Elyahoodayan, S., Larson, C., Cobo, A. M., Meng, E., and Song, D. (2020). Acute in vivo testing of a polymer cuff electrode with integrated microfluidic channels for stimulation, recording, and drug delivery on rat sciatic nerve. J. Neurosci. Methods 336:108634. doi: 10.1016/j.jneumeth.2020.108634

Fallahi, H., Zhang, J., Phan, H. P., and Nguyen, N. T. (2019). Flexible microfluidics: fundamentals, recent developments, and applications. Micromachines 10:830. doi: $10.3390 / \mathrm{mi} 10120830$

Fattahi, P., Yang, G., Kim, G., and Abidian, M. R. (2014). A review of organic and inorganic biomaterials for neural interfaces. Adv. Mater. 26, 1846-1885. doi: 10.1002/adma.201304496

Fekete, Z., and Pongrácz, A. (2017). Multifunctional soft implants to monitor and control neural activity in the central and peripheral nervous system: a review. Sens. Actuat. B Chem. 243, 1214-1223. doi: 10.1016/j.snb.2016.12.096

Ferlauto, L., D’Angelo, A. N., Vagni, P., Leccardi, M. J. I. A., Mor, F. M., Cuttaz, E. A., et al. (2018). Development and characterization of PEDOT:PSS/alginate soft microelectrodes for application in neuroprosthetics. Front. Neurosci. 12:648. doi: $10.3389 /$ fnins.2018.00648

Ferrara, N., Gerber, H. P., and LeCouter, J. (2003). The biology of VEGF and its receptors. Nat. Med. 9, 669-676. doi: 10.1038/nm0603-669

Ferreri, F., Ponzo, D., Vollero, L., Guerra, A., Di Pino, G., Petrichella, S., et al. (2014). Does an intraneural interface short-term implant for robotic hand control modulate sensorimotor cortical integration? An EEG-TMS coregistration study on a human amputee. Restor. Neurol. Neurosci. 32, 281-292. doi: $10.3233 /$ RNN- 130347
Fink, J., Fuhrmann, R., Scharnweber, T., and Franke, R. P. (2008). Stimulation of monocytes and macrophages: possible influence of surface roughness. Clin. Hemorheol. Microcirc. 39, 205-212. doi: 10.3233/ch-2008-1090

Freeberg, M. J., Pinault, G. C. J., Tyler, D. J., Triolo, R. J., and Ansari, R. (2020). Chronic nerve health following implantation of femoral nerve cuff electrodes. J. Neuroeng. Rehabil. 17:95. doi: 10.1186/s12984-020-00720-3

Gamboa, J. R., Mohandes, S., Tran, P. L., Slepian, M. J., and Yoon, J. Y. (2013). Linear fibroblast alignment on sinusoidal wave micropatterns. Colloids Surf. B Biointerf. 104, 318-325. doi: 10.1016/j.colsurfb.2012.11.035

Ganji, M., Hossain, L., Tanaka, A., Thunemann, M., Halgren, E., Gilja, V., et al. (2018). Monolithic and scalable au nanorod substrates improve PEDOT-metal adhesion and stability in neural electrodes. Adv. Healthc. Mater. 7:923. doi: 10.1002/adhm.201800923

Gao, K., Li, G., Liao, L., Cheng, J., Zhao, J., and Xu, Y. (2013). Fabrication of flexible microelectrode arrays integrated with microfluidic channels for stable neural interfaces. Sens. Actuat. A Phys. 197, 9-14. doi: 10.1016/j.sna.2013.04.005

Garde, K., Keefer, E., Botterman, B., Galvan, P., and Romero-Ortega, M. I. (2009). Early interfaced neural activity from chronic amputated nerves. Front. Neuroeng. 2:5. doi: 10.3389/neuro.16.005.2009

Geddes, L. A., and Roeder, R. (2003). Criteria for the selection of materials for implanted electrodes. Ann. Biomed. Eng. 31, 879-890. doi: 10.1114/1.1581292

Gelmi, A., Cieslar-Pobuda, A., de Muinck, E., Los, M., Rafat, M., and Jager, E. W. H. (2016). Direct mechanical stimulation of stem cells: a beating electromechanically active scaffold for cardiac tissue engineering. Adv. Healthc. Mater. 5, 1471-1480. doi: 10.1002/adhm.201600307

Gerwig, R., Fuchsberger, K., Schroeppel, B., Link, G. S., Heusel, G., Kraushaar, U., et al. (2012). PEDOT-CNT composite microelectrodes for recording and electrostimulation applications: fabrication, morphology, and electrical properties. Front. Neuroeng. 5:8. doi: 10.3389/fneng.2012.00008

Gilletti, A., and Muthuswamy, J. (2006). Brain micromotion around implants in the rodent somatosensory cortex. J. Neural Eng. 3, 189-195. doi: 10.1088/17412560/3/3/001

Gilmour, A. D., Green, R. A., and Thomson, C. E. (2013). A low-maintenance, primary cell culture model for the assessment of carbon nanotube toxicity. Toxicol. Environ. Chem. 95, 1129-1144. doi: 10.1080/02772248.2013.844429

Gori, M., Trombetta, M., Santini, D., and Rainer, A. (2015). Tissue engineering and microRNAs: future perspectives in regenerative medicine. Expert Opin. Biol. Ther. 15, 1601-1622. doi: 10.1517/14712598.2015.1071349

Green, R. A., Hassarati, R. T., Goding, J. A., Baek, S., Lovell, N. H., Martens, P. J., et al. (2012). Conductive hydrogels: mechanically robust hybrids for use as biomaterials. Macromol. Biosci. 12, 494-501. doi: 10.1002/mabi.201100490

Green, R. A., Lovell, N. H., and Poole-Warren, L. A. (2009). Cell attachment functionality of bioactive conducting polymers for neural interfaces. Biomaterials 30, 3637-3644. doi: 10.1016/j.biomaterials.2009.03.043

Green, R. A., Lovell, N. H., Wallace, G. G., and Poole-Warren, L. A. (2008). Conducting polymers for neural interfaces: challenges in developing an effective long-term implant. Biomaterials 29, 3393-3399. doi: 10.1016/j.biomaterials. 2008.04.047

Green, R. A., Matteucci, P. B., Hassarati, R. T., Giraud, B., Dodds, C. W., Chen, S., et al. (2013). Performance of conducting polymer electrodes for stimulating neuroprosthetics. J. Neural Eng. 10:016009. doi: 10.1088/17412560/10/1/016009

Guadarrama-Santana, A., and Garcia-Valenzuela, A. (2007). Determination of thickness and dielectric constant of coatings from capacitance measurements. IEEE Instrum. Measur. Mag. 10, 26-31. doi: 10.1109/MIM.2007.4343564

Guo, L., Ma, M., Zhang, N., Langer, R., and Anderson, D. G. (2014). Stretchable polymeric multielectrode array for conformal neural interfacing. Adv. Mater. 26, 1427-1433. doi: 10.1002/adma.201304140

Gutowski, S. M., Shoemaker, J. T., Templeman, K. L., Wei, Y., Latour, R. A., Bellamkonda, R. V., et al. (2015). Protease-degradable PEG-maleimide coating with on-demand release of IL-1Ra to improve tissue response to neural electrodes. Biomaterials 44, 55-70. doi: 10.1016/j.biomaterials.2014.12.009

Hancock, W. W., Gao, W., Faia, K. L., and Csizmadia, V. (2000). Chemokines and their receptors in allograft rejection. Curr. Opin. Immunol. 12, 511-516. doi: 10.1016/S0952-7915(00)00130-8

Hassler, C., Guy, J., Nietzschmann, M., Plachta, D. T. T., Staiger, J. F., and Stieglitz, T. (2016). Intracortical polyimide electrodes with a bioresorbable coating. Biomed. Microdev. 18:81. doi: 10.1007/s10544-016-0106-7 
Heo, D. N., Song, S. J., Kim, H. J., Lee, Y. J., Ko, W. K., Lee, S. J., et al. (2016). Multifunctional hydrogel coatings on the surface of neural cuff electrode for improving electrode-nerve tissue interfaces. Acta Biomater. 39, 25-33. doi: 10.1016/j.actbio.2016.05.009

Hosseini-Nassab, N., Samanta, D., Abdolazimi, Y., Annes, J. P., and Zare, R. N. (2017). Electrically controlled release of insulin using polypyrrole nanoparticles. Nanoscale 9, 143-149. doi: 10.1039/c6nr08288b

Hu, Y., Yang, G., Liang, B., Fang, L., Ma, G., Zhu, Q., et al. (2015). The fabrication of superlow protein absorption zwitterionic coating by electrochemically mediated atom transfer radical polymerization and its application. Acta Biomater. 13, 142-149. doi: 10.1016/j.actbio.2014.11.023

Hulander, M., Lundgren, A., Faxälv, L., Lindahl, T. L., Palmquist, A., Berglin, M., et al. (2013). Gradients in surface nanotopography used to study platelet adhesion and activation. Colloids Surf. B Biointerf. 110, 261-269. doi: 10.1016/j. colsurfb.2013.04.010

Hunt, J. A., Flanagan, B. F., McLaughlin, P. J., Strickland, I., and Williams, D. F. (1996). Effect of biomaterial surface charge on the inflammatory response: evaluation of cellular infiltration and TNF $\alpha$ production. J. Biomed. Mater. Res. 31, 139-144. doi: 10.1002/(sici)1097-4636(199605)31:1<139::aid-jbm15>3.0. $\operatorname{co} ; 2-\mathrm{i}$

Isaksson, J., Kjäll, P., Nilsson, D., Robinson, N., Berggren, M., and RichterDahlfors, A. (2007). Electronic control of Ca2+ signalling in neuronal cells using an organic electronic ion pump. Nat. Mater. 6, 673-679. doi: 10.1038/ nmat 1963

Jayamani, E., Hamdan, S., Rahman, R., and Bakri, M. K. B. (2014). Comparative Study of dielectric properties of hybrid natural fiber composites. Proc. Eng. 97, 536-544. doi: 10.1016/j.proeng.2014.12.280

Jenney, C. R., and Anderson, J. M. (2000). Adsorbed serum proteins responsible for surface dependent human macrophage behavior. J. Biomed. Mater. Res. 49, 435-447. doi: 10.1002/(sici)1097-4636(20000315)49:4<435::aid-jbm2>3.0.co; $2-\mathrm{y}$

Jhaveri, S. J., Hynd, M. R., Dowell-Mesfin, N., Turner, J. N., Shain, W., and Ober, C. K. (2009). Release of nerve growth factor from HEMA hydrogel-coated substrates and its effect on the differentiation of neural cells. Biomacromolecules 10, 174-183. doi: 10.1021/bm801101e

Ji, B., Guo, Z., Wang, M., Yang, B., Wang, X., Li, W., et al. (2018). Flexible polyimide-based hybrid opto-electric neural interface with 16 channels of micro-LEDs and electrodes. Microsyst. Nanoeng. 4:27. doi: 10.1038/s41378-0180027-0

Jiang, S., and Cao, Z. (2010). Ultralow-fouling, functionalizable, and hydrolyzable zwitterionic materials and their derivatives for biological applications. $A d v$. Mater. 22, 920-932. doi: 10.1002/adma.200901407

Jonsson, A., Song, Z., Nilsson, D., Meyerson, B. A., Simon, D. T., Linderoth, B., et al. (2015). Therapy using implanted organic bioelectronics. Sci. Adv. 1:e1500039. doi: $10.1126 /$ sciadv.1500039

Kagan, V. E., Tyurina, Y. Y., Tyurin, V. A., Konduru, N. V., Potapovich, A. I., Osipov, A. N., et al. (2006). Direct and indirect effects of single walled carbon nanotubes on RAW 264.7 macrophages: role of iron. Toxicol. Lett. 165, 88-100. doi: 10.1016/j.toxlet.2006.02.001

Kałuża, D., Jaworska, E., Mazur, M., Maksymiuk, K., and Michalska, A. (2019). Multiwalled Carbon Nanotubes-Poly(3-octylthiophene-2,5-diyl) nanocomposite transducer for ion-selective electrodes: raman spectroscopy insight into the transducer/Membrane interface. Anal. Chem. 91, 9010-9017. doi: 10.1021/acs.analchem.9b01286

Kang, Y. N., Chou, N., Jang, J. W., Byun, D., Kang, H., Moon, D. J., et al. (2019). An intrafascicular neural interface with enhanced interconnection for recording of peripheral nerve signals. IEEE Trans. Neural Syst. Rehabil. Eng. 27, 1312-1319. doi: 10.1109/TNSRE.2019.2917916

Kim, D. H., and Martin, D. C. (2006). Sustained release of dexamethasone from hydrophilic matrices using PLGA nanoparticles for neural drug delivery. Biomaterials 27, 3031-3037. doi: 10.1016/j.biomaterials.2005.12.021

Kim, Y. K., Que, R., Wang, S. W., and Liu, W. F. (2014). Modification of biomaterials with a self-protein inhibits the macrophage response. Adv. Healthc. Mater. 3, 989-994. doi: 10.1002/adhm.201300532

Kleber, C., Bruns, M., Lienkamp, K., Rühe, J., and Asplund, M. (2017). An interpenetrating, microstructurable and covalently attached conducting polymer hydrogel for neural interfaces. Acta Biomater. 58, 365-375. doi: 10. 1016/j.actbio.2017.05.056
Kleber, C., Lienkamp, K., Rühe, J., and Asplund, M. (2019). electrochemically controlled drug release from a conducting polymer hydrogel (PDMAAp/PEDOT) for local therapy and bioelectronics. Adv. Healthc. Mater. 8:1801488. doi: 10.1002/adhm.201801488

Knop, K., Hoogenboom, R., Fischer, D., and Schubert, U. S. (2010). Poly(ethylene glycol) in drug delivery: pros and cons as well as potential alternatives. Angew. Chemie Int. Edn. 49, 6288-6308. doi: 10.1002/anie.200902672

Kotov, N. A., Winter, J. O., Clements, I. P., Jan, E., Timko, B. P., Campidelli, S., et al. (2009). Nanomaterials for neural interfaces. Adv. Mater. 21, 3970-4004. doi: 10.1002/adma.200801984

Kozai, T. D. Y. (2018). The history and horizons of microscale neural interfaces. Micromachines 9:445. doi: 10.3390/mi9090445

Krishnan, A., Dujardin, E., and Ebbesen, T. (1998). Young's modulus of singlewalled nanotubes. Phys. Rev. B Condens. Matter Mater. Phys. 58, 14013-14019. doi: 10.1103/PhysRevB.58.14013

Kumar, S. S., Baker, M. S., Okandan, M., and Muthuswamy, J. (2020). Engineering microscale systems for fully autonomous intracellular neural interfaces. Microsyst. Nanoeng. 6:1. doi: 10.1038/s41378-019-0121-y

Kwon, I. S., Kim, Y. J., Klosterman, L., Forssell, M., Fedder, G. K., and Bettinger, C. J. (2016). In vitro electrochemical characterization of polydopamine melanin as a tissue stimulating electrode material. J. Mater. Chem. B 4, 3031-3036. doi: $10.1039 / \mathrm{c} 5$ tb02618k

Kyriakides, T. R., Foster, M. J., Keeney, G. E., Tsai, A., Giachelli, C. M., ClarkLewis, I., et al. (2004). The CC chemokine ligand, CCL2/MCP1, participates in macrophage fusion and foreign body giant cell formation. Am. J. Pathol. 165, 2157-2166. doi: 10.1016/S0002-9440(10)63265-8

Lago, N., Yoshida, K., Koch, K. P., and Navarro, X. (2007). Assessment of biocompatibility of chronically implanted polyimide and platinum intrafascicular electrodes. IEEE Trans. Biomed. Eng. 54, 281-290. doi: 10.1109/ TBME.2006.886617

Lam, J. K. W., Chow, M. Y. T., Zhang, Y., and Leung, S. W. S. (2015). siRNA versus miRNA as therapeutics for gene silencing. Mol. Ther. Nucleic Acids 4:e252. doi: $10.1038 / \mathrm{mtna} .2015 .23$

Leach, J. B., Achyuta, A. K. H., and Murthy, S. K. (2010). Bridging the divide between neuroprosthetic design, tissue engineering and neurobiology. Front. Neuroeng. 2:18. doi: 10.3389/neuro.16.018.2009

Leask, A., Holmes, A., and Abraham, D. J. (2002). Connective tissue growth factor: a new and important player in the pathogenesis of fibrosis. Curr. Rheumatol. Rep. 4, 136-142. doi: 10.1007/s11926-002-0009-x

Lecomte, A., Degache, A., Descamps, E., Dahan, L., and Bergaud, C. (2017). In vitro and in vivo biostability assessment of chronically-implanted Parylene C neural sensors. Sens. Actuat. B Chem. 251, 1001-1008. doi: 10.1016/j.snb.2017.05.057

Lee, D. H., Yun, H. D., Jung, E. D., Chu, J. H., Nam, Y. S., Song, S., et al. (2019). Ultrathin Graphene Intercalation in PEDOT:PSS/colorless polyimidebased transparent electrodes for enhancement of optoelectronic performance and operational stability of organic devices. ACS Appl. Mater. Interf. 11, 2106921077. doi: 10.1021/acsami.9b04118

Lee, K. K., He, J., Singh, A., Massia, S., Ehteshami, G., Kim, B., et al. (2004). Polyimide-based intracortical neural implant with improved structural stiffness. J. Micromech. Microeng. 14, 32-37. doi: 10.1088/0960-1317/14/1/305

Lee, K. Y., and Mooney, D. J. (2012). Alginate: properties and biomedical applications. Prog. Polym. Sci. 37, 106-126. doi: 10.1016/j.progpolymsci.2011. 06.003

Lee, Y. J., Kim, H. J., Kang, J. Y., Do, S. H., and Lee, S. H. (2017). Biofunctionalization of nerve interface via biocompatible polymer-roughened pt black on cuff electrode for chronic recording. Adv. Healthc. Mater. 6:1601022. doi: 10.1002/adhm.201601022

Lim, F., and Sun, A. M. (1980). Microencapsulated islets as bioartificial endocrine pancreas. Science 210, 908-910. doi: 10.1126/science.6776628

Lim, H. R., Kim, Y. S., Kwon, S., Mahmood, M., Kwon, Y. T., Lee, Y., et al. (2020). Wireless, flexible, ion-selective electrode system for selective and repeatable detection of sodium. Sensors 20, 1-11. doi: 10.3390/s20113297

Lishko, V. K., Podolnikova, N. P., Yakubenko, V. P., Yakovlev, S., Medved, L., Yadav, S. P., et al. (2004). Multiple binding sites in fibrinogen for integrin $\alpha \mathrm{M} \beta$ 2 (Mac-1). J. Biol. Chem. 279, 44897-44906. doi: 10.1074/jbc.M408012200

Löffler, S., Seyock, S., Nybom, R., Jacobson, G. B., and Richter-Dahlfors, A. (2016). Electrochemically triggered release of acetylcholine from scCO2 impregnated conductive polymer films evokes intracellular $\mathrm{Ca} 2+$ signaling in neurotypic 
SH-SY5Y cells. J. Control. Release 243, 283-290. doi: 10.1016/j.jconrel.2016.10. 020

Lorach, H., Goetz, G., Smith, R., Lei, X., Mandel, Y., Kamins, T., et al. (2015). Photovoltaic restoration of sight with high visual acuity. Nat. Med. 21, 476-482. doi: $10.1038 / \mathrm{nm} .3851$

Lotti, F., Ranieri, F., Vadalà, G., Zollo, L., and Di Pino, G. (2017). Invasive intraneural interfaces: foreign body reaction issues. Front. Neurosci. 11:497. doi: 10.3389/fnins.2017.00497

Lucke, S., Walschus, U., Hoene, A., Schnabelrauch, M., Nebe, J. B., Finke, B., et al. (2018). The in vivo inflammatory and foreign body giant cell response against different poly(l-lactide-co-d/l-lactide) implants is primarily determined by material morphology rather than surface chemistry. J. Biomed. Mater. Res. Part A 106, 2726-2734. doi: 10.1002/jbm.a.36500

Luo, X., Matranga, C., Tan, S., Alba, N., and Cui, X. T. (2011). Carbon nanotube nanoreservior for controlled release of anti-inflammatory dexamethasone. Biomaterials 32, 6316-6323. doi: 10.1016/j.biomaterials.2011.05.020

Luttikhuizen, D. T., Harmsen, M. C., and Van Luyn, M. J. A. (2006). Cellular and molecular dynamics in the foreign body reaction. Tissue Eng. 12, 1955-1970. doi: 10.1089/ten.2006.12.1955

Lynn, A. D., Blakney, A. K., Kyriakides, T. R., and Bryant, S. J. (2011). Temporal progression of the host response to implanted poly(ethylene glycol)-based hydrogels. J. Biomed. Mater. Res. Part A 96, 621-631. doi: 10.1002/jbm.a.33015

Ma, M., Liu, W. F., Hill, P. S., Bratlie, K. M., Siegwart, D. J., Chin, J., et al. (2011). Development of cationic polymer coatings to regulate foreign-body responses. Adv. Mater. 23, H189-H194. doi: 10.1002/adma.201100513

Madden, L. R., Mortisen, D. J., Sussman, E. M., Dupras, S. K., Fugate, J. A., Cuy, J. L., et al. (2010). Proangiogenic scaffolds as functional templates for cardiac tissue engineering. Proc. Natl. Acad. Sci. U. S. A. 107, 15211-15216. doi: 10.1073/pnas.1006442107

Mandal, H. S., Kastee, J. S., McHail, D. G., Rubinson, J. F., Pancrazio, J. J., and Dumas, T. C. (2015). Improved Poly(3,4-Ethylenedioxythiophene) (PEDOT) for neural stimulation. Neuromodulation 18, 657-663. doi: 10.1111/ner.12285

Mario Cheong, G. L., Lim, K. S., Jakubowicz, A., Martens, P. J., Poole-Warren, L. A., and Green, R. A. (2014). Conductive hydrogels with tailored bioactivity for implantable electrode coatings. Acta Biomater. 10, 1216-1226. doi: 10.1016/ j.actbio.2013.12.032

Matlaga, B. F., Yasenchak, L. P., and Salthouse, T. N. (1976). Tissue response to implanted polymers: the significance of sample shape. J. Biomed. Mater. Res. 10, 391-397. doi: 10.1002/jbm.820100308

McConnell, G. C., Rees, H. D., Levey, A. I., Gutekunst, C. A., Gross, R. E., and Bellamkonda, R. V. (2009). Implanted neural electrodes cause chronic, local inflammation that is correlated with local neurodegeneration. J. Neural Eng. 6, 056003. doi: 10.1088/1741-2560/6/5/056003

Mercanzini, A., Reddy, S. T., Velluto, D., Colin, P., Maillard, A., Bensadoun, J. C., et al. (2010). Controlled release nanoparticle-embedded coatings reduce the tissue reaction to neuroprostheses. J. Control. Release 145, 196-202. doi: 10.1016/j.jconrel.2010.04.025

Merrill, D. R., Bikson, M., and Jefferys, J. G. R. (2005). Electrical stimulation of excitable tissue: design of efficacious and safe protocols. J. Neurosci. Methods 141, 171-198. doi: 10.1016/j.jneumeth.2004.10.020

Metz, S., Bertsch, A., Bertrand, D., and Renaud, P. (2004). Flexible polyimide probes with microelectrodes and embedded microfluidic channels for simultaneous drug delivery and multi-channel monitoring of bioelectric activity. Biosens. Bioelectron. 19, 1309-1318. doi: 10.1016/j.bios.2003. 11.021

Miele, E., Spinelli, G. P., Miele, E., Fabrizio, E., Di Ferretti, E., Tomao, S., et al. (2012). Nanoparticle-based delivery of small interfering RNA: challenges for cancer therapy. Int. J. Nanomed. 7, 3637-3657. doi: 10.2147/IJN.S23696

Minev, I. R., Musienko, P., Hirsch, A., Barraud, Q., Wenger, N., Moraud, E. M., et al. (2015). Electronic dura mater for long-term multimodal neural interfaces. Science 347, 159-163. doi: 10.1126/science. 1260318

Morrison, T. J., Sefton, E., Marquez-Chin, M., Popovic, M. R., Morshead, C. M., and Naguib, H. E. (2019). A 3D printed device for low cost neural stimulation in mice. Front. Neurosci. 13:784. doi: 10.3389/fnins.2019.00784

Mueller, M., De La Oliva, N., Del Valle, J., Delgado-Martinez, I., Navarro, X., and Stieglitz, T. (2017). Rapid prototyping of flexible intrafascicular electrode arrays by picosecond laser structuring. J. Neural Eng. 14:066016. doi: 10.1088/17412552/aa7eea
Navarro, X., Krueger, T. B., Lago, N., Micera, S., Stieglitz, T., and Dario, P. (2005). A critical review of interfaces with the peripheral nervous system for the control of neuroprostheses and hybrid bionic systems. J. Peripher. Nerv. Syst. 10, 229-258. doi: 10.1111/j.1085-9489.2005.10303.x

Navarro, X., Valderrama, E., Stieglitz, T., and Schüttler, M. (2001). Selective fascicular stimulation of the rat sciatic nerve with multipolar polyimide cuff electrodes. Restor. Neurol. Neurosci. 18, 9-21.

Nguyen, J. K., Park, D. J., Skousen, J. L., Hess-Dunning, A. E., Tyler, D. J., Rowan, S. J., et al. (2014). Mechanically-compliant intracortical implants reduce the neuroinflammatory response. J. Neural Eng. 11:056014. doi: 10.1088/17412560/11/5/056014

Oddo, C. M., Raspopovic, S., Artoni, F., Mazzoni, A., Spigler, G., Petrini, F., et al. (2016). Intraneural stimulation elicits discrimination of textural features by artificial fingertip in intact and amputee humans. eLife 5:e09148. doi: 10.7554/ eLife.09148

Omar, R., Yang, J., Liu, H., Davies, N. M., and Gong, Y. (2016). Hepatic stellate cells in liver fibrosis and sirna-based therapy. Rev. Physiol. Biochem. Pharmacol. 172, 1-37. doi: 10.1007/112_2016_6

Ono, S. J., Nakamura, T., Miyazaki, D., Ohbayashi, M., Dawson, M., and Toda, M. (2003). Chemokines: roles in leukocyte development, trafficking, and effector function. J. Allergy Clin. Immunol. 111, 1185-1199. doi: 10.1067/mai.2003.1594

Ordonez, J. (2012). Thin films and microelectrode arrays for neuroprosthetics ProQuest. MRS Bull. Suppl. Mater. Neural Interf. 37, 590-598. doi: 10.1557/mrs. 2012.117

Ortiz-Catalan, M., Marin-Millan, J., Delbeke, J., Håkansson, B., and Brånemark, R. (2013). Effect on signal-to-noise ratio of splitting the continuous contacts of cuff electrodes into smaller recording areas. J. Neuroeng. Rehabil. 10:22. doi: 10.1186/1743-0003-10-22

Ostuni, E., Chapman, R. G., Holmlin, R. E., Takayama, S., and Whitesides, G. M. (2001). A survey of structure-property relationships of surfaces that resist the adsorption of protein. Langmuir 17, 5605-5620. doi: 10.1021/la010384m

Polikov, V. S., Tresco, P. A., and Reichert, W. M. (2005). Response of brain tissue to chronically implanted neural electrodes. J. Neurosci. Methods 148, 1-18. doi: 10.1016/j.jneumeth.2005.08.015

Proctor, C. M., Slézia, A., Kaszas, A., Ghestem, A., del Agua, I., Pappa, A. M., et al. (2018). Electrophoretic drug delivery for seizure control. Sci. Adv. 4:aau1291. doi: 10.1126/sciadv.aau1291

Qi, D., Liu, Z., Liu, Y., Jiang, Y., Leow, W. R., Pal, M., et al. (2017). Highly stretchable, compliant, polymeric microelectrode arrays for in vivo electrophysiological interfacing. Adv. Mater. 29:1702800. doi: 10.1002/adma. 201702800

Qin, D., Xia, Y., and Whitesides, G. M. (2010). Soft lithography for micro- and nanoscale patterning. Nat. Protoc. 5, 491-502. doi: 10.1038/nprot.2009.234

Raffa, V., Pensabene, V., Menciassi, A., and Dario, P. (2007). Design criteria of neuron/electrode interface. The focused ion beam technology as an analytical method to investigate the effect of electrode surface morphology on neurocompatibility. Biomed. Microdev. 9, 371-383. doi: 10.1007/s10544-0069042-2

Rao, S. S., Han, N., and Winter, J. O. (2011). Polylysine-modified PEG-based hydrogels to enhance the neuro-electrode interface. J. Biomater. Sci. Polym. Edn. 22, 611-625. doi: 10.1163/092050610X488241

Raspopovic, S., Capogrosso, M., Petrini, F. M., Bonizzato, M., Rigosa, J., Di Pino, G., et al. (2014). Bioengineering: restoring natural sensory feedback in realtime bidirectional hand prostheses. Sci. Transl. Med. 6:222ra19. doi: 10.1126/ scitranslmed. 3006820

Ratner, B. D. (2002). Reducing capsular thickness and enhancing angiogenesis around implant drug release systems. J. Control. Release 78, 211-218. doi: 10.1016/S0168-3659(01)00502-8

Retterer, S. T., Smith, K. L., Bjornsson, C. S., Neeves, K. B., Spence, A. J. H., Turner, J. N., et al. (2004). Model neural prostheses with integrated microfluidics: a potential intervention strategy for controlling reactive cell and tissue responses. IEEE Trans. Biomed. Eng. 51, 2063-2073. doi 10.1109/TBME.2004.8 34288

Richardson, D. L., Pepper, D. S., and Kay, A. B. (1976). Chemotaxis for human monocytes by fibrinogen-derived peptides. Br. J. Haematol. 32, 507-514. doi: 10.1111/j.1365-2141.1976.tb00953.x

Rodriguez, P. L., Harada, T., Christian, D. A., Pantano, D. A., Tsai, R. K., and Discher, D. E. (2013). Minimal "self" peptides that inhibit phagocytic clearance 
and enhance delivery of nanoparticles. Science 339, 971-975. doi: 10.1126/ science. 1229568

Rombaut, J., Fernandez, M., Mazumder, P., and Pruneri, V. (2019). Nanostructured hybrid-material transparent surface with antireflection properties and a facile fabrication process. ACS Omega 4, 19840-19846. doi: 10.1021/acsomega. $9 \mathrm{~b} 02775$

Rossini, P. M., Micera, S., Benvenuto, A., Carpaneto, J., Cavallo, G., Citi, L., et al. (2010). Double nerve intraneural interface implant on a human amputee for robotic hand control. Clin. Neurophysiol. 121, 777-783. doi: 10.1016/j.clinph. 2010.01.001

Rousche, P. J., Pellinen, D. S., Pivin, D. P., Williams, J. C., Vetter, R. J., and Kipke, D. R. (2001). Flexible polyimide-based intracortical electrode arrays with bioactive capability. IEEE Trans. Biomed. Eng. 48, 361-370. doi: 10.1109/10. 914800

Rubehn, B., and Stieglitz, T. (2010). In vitro evaluation of the long-term stability of polyimide as a material for neural implants. Biomaterials 31, 3449-3458. doi: 10.1016/j.biomaterials.2010.01.053

Saeed, M., Ren, W., and Wu, A. (2018). Therapeutic applications of iron oxide based nanoparticles in cancer: basic concepts and recent advances. Biomater. Sci. 6, 708-725. doi: 10.1039/c7bm00999b

Salazar-Montes, A. M., Hernández-Ortega, L. D., Lucano-Landeros, M. S., and Armendariz-Borunda, J. (2015). New gene therapy strategies for hepatic fibrosis. World J. Gastroenterol. 21, 3813-3825. doi 10.3748/wjg.v21.i 13.3813

Salthouse, T. N. (1984). Some aspects of macrophage behavior at the implant interface. J. Biomed. Mater. Res. 18, 395-401. doi: 10.1002/jbm.820180407

Samba, R., Herrmann, T., and Zeck, G. (2015). PEDOT - CNT coated electrodes stimulate retinal neurons at low voltage amplitudes and low charge densities. J. Neural Eng. 12, 480-488. doi: 10.1088/1741-2560/12/1/016014

Sergi, P. N., del Oliva Valle, N. J., de la Micera, S., and Navarro, X. (2020). A datadriven polynomial approach to reproduce the scar tissue outgrowth around neural implants. J. Mater. Sci. Mater. Med. 31:59. doi: 10.1007/s10856-02006396-4

Serino, A., Akselrod, M., Salomon, R., Martuzzi, R., Blefari, M. L., Canzoneri, E., et al. (2017). Upper limb cortical maps in amputees with targeted muscle and sensory reinnervation. Brain 140, 2993-3011. doi: 10.1093/brain/awx242

Sharifi, F., Htwe, S. S., Righi, M., Liu, H., Pietralunga, A., Yesil-Celiktas, O., et al. (2019). A Foreign body response-on-a-chip platform. Adv. Healthc. Mater. 8:e1801425. doi: 10.1002/adhm.201801425

Simon, D. T., Kurup, S., Larsson, K. C., Hori, R., Tybrandt, K., Goiny, M., et al. (2009). Organic electronics for precise delivery of neurotransmitters to modulate mammalian sensory function. Nat. Mater. 8, 742-746. doi: 10.1038/ nmat 2494

Sin, M. C., Chen, S. H., and Chang, Y. (2014a). Hemocompatibility of zwitterionic interfaces and membranes. Polym. J. 46, 436-443. doi: 10.1038/pj.2014.46

Sin, M. C., Sun, Y. M., and Chang, Y. (2014b). Zwitterionic-based stainless steel with well-defined polysulfobetaine brushes for general bioadhesive control. ACS Appl. Mater. Interf. 6, 861-873. doi: 10.1021/am404 1256

Smiley, S. T., King, J. A., and Hancock, W. W. (2001). Fibrinogen stimulates macrophage chemokine secretion through toll-like receptor 4. J. Immunol. 167, 2887-2894. doi: 10.4049/jimmunol.167.5.2887

Smith, R. S., Zhang, Z., Bouchard, M., Li, J., Lapp, H. S., Brotske, G. R., et al. (2012). Vascular catheters with a nonleaching poly-sulfobetaine surface modification reduce thrombus formation and microbial attachment. Sci. Transl. Med. 4:153ra132. doi: 10.1126/scitranslmed.30 04120

Sohal, H. S., Jackson, A., Jackson, R., Clowry, G. J., Vassilevski, K., O’Neill, A., et al. (2014). The sinusoidal probe: a new approach to improve electrode longevity. Front. Neuroeng. 7:10. doi: 10.3389/fneng.2014.00010

Soltanzadeh, R., Afsharipour, E., Anssari, N., Mansouri, B., and Shafai, C. (2020). Structural and performance comparison between SU-8 microfabricated and 3D-printed microneedle electrodes. J. 3D Print. Med. 4, 29-44. doi: 10.2217/ 3dp-2019-0020

Spataro, L., Dilgen, J., Retterer, S., Spence, A. J., Isaacson, M., Turner, J. N., et al. (2005). Dexamethasone treatment reduces astroglia responses to inserted neuroprosthetic devices in rat neocortex. Exp. Neurol. 194, 289-300. doi: 10. 1016/j.expneurol.2004.08.037
Spencer, K. C., Sy, J. C., Ramadi, K. B., Graybiel, A. M., Langer, R., and Cima, M. J. (2017). Characterization of mechanically matched hydrogel coatings to improve the biocompatibility of neural implants. Sci. Rep. 7:1952. doi: 10.1038/s41598017-02107-2

Staples, N. A., Goding, J. A., Gilmour, A. D., Aristovich, K. Y., Byrnes-Preston, P., Holder, D. S., et al. (2018). Conductive hydrogel electrodes for delivery of longterm high frequency pulses. Front. Neurosci. 11:748. doi: 10.3389/fnins.2017. 00748

Stubbs, E. B. (2020). Targeting the blood-nerve barrier for the management of immune-mediated peripheral neuropathies. Exp. Neurol. 331:113385. doi: 10. 1016/j.expneurol.2020.113385

Surendran, S. P., Thomas, R. G., Moon, M. J., and Jeong, Y. Y. (2017). Nanoparticles for the treatment of liver fibrosis. Int. J. Nanomed. 12, 6997-7006. doi: 10.2147/ IJN.S145951

Svennersten, K., Berggren, M., Richter-Dahlfors, A., and Jager, E. W. H. (2011). Mechanical stimulation of epithelial cells using polypyrrole microactuators. $\mathrm{Lab}$ Chip 11, 3287-3293. doi: 10.1039/c1lc20436j

Szaba, F. M., and Smiley, S. T. (2002). Roles for thrombin and fibrin(ogen) in cytokine/chemokine production and macrophage adhesion in vivo. Blood 99, 1053-1059. doi: 10.1182/blood.V99.3.1053

Szarowski, D. H., Andersen, M. D., Retterer, S., Spence, A. J., Isaacson, M., Craighead, H. G., et al. (2003). Brain responses to micro-machined silicon devices. Brain Res. 983, 23-35. doi: 10.1016/S0006-8993(03)03023-3

Szostak, K. M., Grand, L., and Constandinou, T. G. (2017). Neural interfaces for intracortical recording: requirements, fabrication methods, and characteristics. Front. Neurosci. 11:665. doi: 10.3389/fnins.2017.00665

Takehara, H., Nagaoka, A., Noguchi, J., Akagi, T., Kasai, H., and Ichiki, T. (2014). Lab-on-a-brain: implantable micro-optical fluidic devices for neural cell analysis in vivo. Sci. Rep. 4:6721. doi: 10.1038/srep06721

Tang, L., and Eaton, J. W. (1993). Fibrin(ogen) mediates acute inflammatory responses to biomaterials. J. Exp. Med. 178, 2147-2156. doi: 10.1084/jem.178. 6.2147

Tang, L., Jennings, T. A., and Eaton, J. W. (1998). Mast cells mediate acute inflammatory responses to implanted biomaterials. Proc. Natl. Acad. Sci. U.S.A. 95, 8841-8846. doi: 10.1073/pnas.95.15.8841

Tao, F., and Kobzik, L. (2002). Lung macrophage-epithelial cell interactions amplify particle-mediated cytokine release. Am. J. Respir. Cell Mol. Biol. 26, 499-505. doi: $10.1165 / \mathrm{ajrcmb} .26 .4 .4749$

Teixeira, A. I., Ilkhanizadeh, S., Wigenius, J. A., Duckworth, J. K., Inganäs, O., and Hermanson, O. (2009). The promotion of neuronal maturation on soft substrates. Biomaterials 30, 4567-4572. doi: 10.1016/j.biomaterials.2009.05.013

Thull, R. (2002). Physicochemical principles of tissue material interactions. Biomolecu. Eng. 19, 43-50. doi: 10.1016/S1389-0344(02)00009-6

Tian, F., Cui, D., Schwarz, H., Estrada, G. G., and Kobayashi, H. (2006). Cytotoxicity of single-wall carbon nanotubes on human fibroblasts. Toxicol. Vitr. 20, 1202-1212. doi: 10.1016/j.tiv.2006.03.008

Trel'Ová, D., Salgarella, A. R., Ricotti, L., Giudetti, G., Cutrone, A., Šrámková, P., et al. (2019). Soft hydrogel zwitterionic coatings minimize fibroblast and macrophage adhesion on polyimide substrates. Langmuir 35, 1085-1099. doi: 10.1021/acs.langmuir.8b00765

Tyler, D. J., and Durand, D. M. (2002). Functionally selective peripheral nerve stimulation with a flat interface nerve electrode. IEEE Trans. Neural Syst. Rehabil. Eng. 10, 294-303. doi: 10.1109/TNSRE.2002.806840

Uguz, I., Proctor, C. M., Curto, V. F., Pappa, A. M., Donahue, M. J., Ferro, M., et al. (2017). A microfluidic ion pump for in vivo drug delivery. Adv. Mater. 29:1701217. doi: 10.1002/adma.201701217

Valle, G., Mazzoni, A., Iberite, F., D’Anna, E., Strauss, I., Granata, G., et al. (2018). Biomimetic intraneural sensory feedback enhances sensation naturalness, tactile sensitivity, and manual dexterity in a bidirectional prosthesis. Neuron 100, 37-45.e7. doi: 10.1016/j.neuron.2018.08.033

Vegas, A. J., Veiseh, O., Gürtler, M., Millman, J. R., Pagliuca, F. W., Bader, A. R., et al. (2016). Long-term glycemic control using polymer-encapsulated human stem cell-derived beta cells in immune-competent mice. Nat. Med. 22, 306-311. doi: $10.1038 / \mathrm{nm} .4030$

Veiseh, O., Doloff, J. C., Ma, M., Vegas, A. J., Tam, H. H., Bader, A. R., et al. (2015). Size- and shape-dependent foreign body immune response to materials implanted in rodents and non-human primates. Nat. Mater. 14, 643-651. doi: $10.1038 /$ nmat 4290 
Vitale, F., Vercosa, D. G., Rodriguez, A. V., Pamulapati, S. S., Seibt, F., Lewis, E., et al. (2018). Fluidic microactuation of flexible electrodes for neural recording. Nano Lett. 18, 326-335. doi: 10.1021/acs.nanolett.7b04184

Von Der Mark, K., Park, J., Bauer, S., and Schmuki, P. (2010). Nanoscale engineering of biomimetic surfaces: cues from the extracellular matrix. Cell Tissue Res. 339, 131-153. doi: 10.1007/s00441-009-0896-5

Wadhwa, R., Lagenaur, C. F., and Cui, X. T. (2006). Electrochemically controlled release of dexamethasone from conducting polymer polypyrrole coated electrode. J. Control. Release 110, 531-541. doi 10.1016/j.jconrel.2005. 10.027

Wang, J., Thow, X. Y., Wang, H., Lee, S., Voges, K., Thakor, N. V., et al. (2018). A highly selective 3D Spiked Ultraflexible Neural (SUN) interface for decoding peripheral nerve sensory information. Adv. Healthc. Mater. 7:987. doi: 10.1002/ adhm.201700987

Wang, M., Mi, G., Shi, D., Bassous, N., Hickey, D., and Webster, T. J. (2018). Nanotechnology and nanomaterials for improving neural interfaces. Adv. Funct. Mater. 28:1700905. doi: 10.1002/adfm.201700905

Ward, W. K. (2008). A review of the foreign-body response to subcutaneouslyimplanted devices: the role of macrophages and cytokines in biofouling and fibrosis. J. Diabetes Sci. Technol. 2, 768-777. doi: 10.1177/193229680800200504

Ward, W. K., Slobodzian, E. P., Tiekotter, K. L., and Wood, M. D. (2002). The effect of microgeometry, implant thickness and polyurethane chemistry on the foreign body response to subcutaneous implants. Biomaterials 23, 4185-4192. doi: 10.1016/S0142-9612(02)00160-6

Ware, T., Simon, D., Rennaker, R. L., and Voit, W. (2013). Smart polymers for neural interfaces. Polym. Rev. 53, 108-129. doi: 10.1080/15583724.2012.751924

Weaver, C. L., Larosa, J. M., Luo, X., and Cui, X. T. (2014). Electrically controlled drug delivery from graphene oxide nanocomposite films. ACS Nano 8, 18341843. doi: $10.1021 / \mathrm{nn} 406223 \mathrm{e}$

Wichterle, O., and Lím, D. (1960). Hydrophilic gels for biological use. Nature 185, 117-118. doi: 10.1038/185117a0

Wilks, S. J., Woolley, A. J., Ouyang, L., Martin, D. C., and Otto, K. J. (2011). In vivo polymerization of poly(3,4-ethylenedioxythiophene) (PEDOT) in rodent cerebral cortex. Annu. Int. Conf. IEEE Eng. Med. Biol. Soc. 2011, 5412-5415. doi: 10.1109/IEMBS.2011.6091338

Winslow, B. D., and Tresco, P. A. (2010). Quantitative analysis of the tissue response to chronically implanted microwire electrodes in rat cortex. Biomaterials 31, 1558-1567. doi: 10.1016/j.biomaterials.2009.11.049

Woeppel, K., Yang, Q., and Cui, X. T. (2017). Recent advances in neural electrodetissue interfaces. Curr. Opin. Biomed. Eng. 4, 21-31. doi: 10.1016/j.cobme.2017. 09.003

Wu, J., Xiao, Z., Chen, A., He, H., He, C., Shuai, X., et al. (2018). Sulfated zwitterionic poly(sulfobetaine methacrylate) hydrogels promote complete skin regeneration. Acta Biomater. 71, 293-305. doi: 10.1016/j.actbio.2018.02.034

Wu, W., He, Q., and Jiang, C. (2008). Magnetic iron oxide nanoparticles: synthesis and surface functionalization strategies. Nanoscale Res. Lett. 3, 397-415. doi: 10.1007/s11671-008-9174-9

Wünsche, J., Cardenas, L., Rosei, F., Cicoira, F., Gauvin, R., Graeff, C. F. O., et al. (2013). In situ formation of dendrites in eumelanin thin films between gold electrodes. Adv. Funct. Mater. 23, 5591-5598. doi: 10.1002/adfm.201300715

Wurth, S., Capogrosso, M., Raspopovic, S., Gandar, J., Federici, G., Kinany, N., et al. (2017). Long-term usability and bio-integration of polyimide-based intra-neural stimulating electrodes. Biomaterials 122, 114-129. doi: 10.1016/j. biomaterials.2017.01.014

Xavier, N., and Jaume, D. V. (2014). Regenerative neural interfaces for neuroprosthetic applications. Front. Neuroeng. 7:3. doi: 10.3389/conf.fneng. 2014.11.00003

Xiao, S., Castro, R., Rodrigues, J., Shi, X., and Tomá, H. (2014). PAMAM dendrimer/pDNA functionalized-magnetic iron oxide nanoparticles for gene delivery. J. Biomed. Nanotechnol. 11, 1418-1430. doi: 10.1166/jbn.2015.2101

Xiao, Y., He, L., and Che, J. (2012). An effective approach for the fabrication of reinforced composite hydrogel engineered with SWNTs, polypyrrole and PEGDA hydrogel. J. Mater. Chem. 22, 8076-8082. doi: 10.1039/c2jm30601h
Xie, X., Rieth, L., Williams, L., Negi, S., Bhandari, R., Caldwell, R., et al. (2014). Long-term reliability of $\mathrm{Al} 2 \mathrm{O} 3$ and Parylene $\mathrm{C}$ bilayer encapsulated utah electrode array based neural interfaces for chronic implantation. J. Neural Eng. 11:026016. doi: 10.1088/1741-2560/11/2/026016

Xu, H., Belkacemi, L., Jog, M., Parrent, A., and Hebb, M. O. (2013). Neurotrophic factor expression in expandable cell populations from brain samples in living patients with Parkinson's disease. FASEB J. 27, 4157-4168. doi: 10.1096/fj.12226555

Yamato, H., Ohwa, M., and Wernet, W. (1995). Stability of polypyrrole and poly(3,4-ethylenedioxythiophene) for biosensor application. J. Electroanal. Chem. 397, 163-170. doi: 10.1016/0022-0728(95)04156-8

Yang, C., and Suo, Z. (2018). Hydrogel ionotronics. Nat. Rev. Mater. 3, 125-142. doi: 10.1038/s41578-018-0018-7

Yang, H., Rahman, M. T., Du, D., Panat, R., and Lin, Y. (2016). 3-D printed adjustable microelectrode arrays for electrochemical sensing and biosensing. Sens. Actuat. B Chem. 230, 600-606. doi: 10.1016/j.snb.2016.02.113

Yesilyurt, V., Veiseh, O., Doloff, J. C., Li, J., Bose, S., Xie, X., et al. (2017). A facile and versatile method to endow biomaterial devices with zwitterionic surface coatings. Adv. Healthc. Mater. 6:1601091. doi: 10.1002/adhm.201601091

Yildiz, K. A., Shin, A. Y., and Kaufman, K. R. (2020). Interfaces with the peripheral nervous system for the control of a neuroprosthetic limb: a review. J. Neuroeng. Rehabil. 17:43. doi: 10.1186/s12984-020-00667-5

Yoshida, K., and Stein, R. B. (1999). Characterization of signals and noise rejection with bipolar longitudinal intrafascicular electrodes. IEEE Trans. Biomed. Eng. 46, 226-234. doi: 10.1109/10.740885

Yu, Q., Xiong, X., Zhao, L., Xu, T., and Wang, Q. (2020). Antifibrotic effects of specific siRNA targeting connective tissue growth factor delivered by polyethyleneimine-functionalized magnetic iron oxide nanoparticles on LX-2 cells. Mol. Med. Rep. 21, 181-190. doi: 10.3892/mmr.2019.10834

Zeglio, E., Rutz, A. L., Winkler, T. E., Malliaras, G. G., and Herland, A. (2019). Conjugated polymers for assessing and controlling biological functions. $A d v$. Mater. 31:1806712. doi: 10.1002/adma.201806712

Zhang, L., Cao, Z., Bai, T., Carr, L., Ella-Menye, J. R., Irvin, C., et al. (2013). Zwitterionic hydrogels implanted in mice resist the foreign-body reaction. Nat. Biotechnol. 31, 553-556. doi: 10.1038/nbt.2580

Zhang, Y., An, D., Pardo, Y., Chiu, A., Song, W., Liu, Q., et al. (2017). High-watercontent and resilient PEG-containing hydrogels with low fibrotic response. Acta Biomater. 53, 100-108. doi: 10.1016/j.actbio.2017.02.028

Zheng, X., Woeppel, K. M., Griffith, A. Y., Chang, E., Looker, M. J., Fisher, L. E., et al. (2019). Soft conducting elastomer for peripheral nerve interface. $A d v$. Healthc. Mater. 8:1311. doi: 10.1002/adhm.201801311

Zhu, Z., Yang, G., Li, R., and Pan, T. (2017). Photopatternable pedot:Pss/peg hybrid thin film with moisture stability and sensitivity. Microsyst. Nanoeng. 3:17004. doi: 10.1038 /micronano.2017.4

Ziegler, D., Suzuki, T., and Takeuchi, S. (2006). Fabrication of flexible neural probes with built-in microfluidic channels by thermal bonding of Parylene. J. Microelectromech. Syst. 15, 1477-1482. doi: 10.1109/JMEMS.2006.879681

Zollo, L., Pino, G. D., Ciancio, A. L., Ranieri, F., Cordella, F., Gentile, C., et al. (2019). Restoring tactile sensations via neural interfaces for real-time forceand-slippage closed-loop control of bionic hands. Sci. Robot. 4:aau9924. doi: $10.1126 /$ scirobotics.aau9924

Conflict of Interest: The authors declare that the research was conducted in the absence of any commercial or financial relationships that could be construed as a potential conflict of interest.

Copyright (C) 2021 Gori, Vadalà, Giannitelli, Denaro and Di Pino. This is an openaccess article distributed under the terms of the Creative Commons Attribution License (CC BY). The use, distribution or reproduction in other forums is permitted, provided the original author(s) and the copyright owner(s) are credited and that the original publication in this journal is cited, in accordance with accepted academic practice. No use, distribution or reproduction is permitted which does not comply with these terms. 